\title{
1 TAOK2 is an ER-localized Kinase that Catalyzes the Dynamic Tethering of
}

2

3

4 mechanism for control of ER dynamics critical for cell growth and division.

Kimya Nourbakhsh ${ }^{1 \#,}$ Amy A. Ferreccio ${ }^{1 \#}$, Matthew J. Bernard ${ }^{1}$ and Smita Yadav ${ }^{1 *}$

\# denotes equal contribution

1. Department of Pharmacology, University of Washington, Seattle, WA 98195

*Corresponding author, smitay@uw.edu

\section{Summary}

The endoplasmic reticulum (ER) depends on extensive association with the microtubule cytoskeleton for its structure, function and mitotic inheritance. The identity of molecular tethers that mediate ER-microtubule coupling, and mechanisms through which dynamic tethering is regulated are poorly understood. Here, we identify, Thousand And One amino acid Kinase 2 (TAOK2) as a pleiotropic protein kinase that mediates tethering of ER to microtubules. We show that TAOK2 is a unique multipass membrane spanning serine/threonine kinase localized in distinct ER domains via four transmembrane and amphipathic helices. Using in vitro and cellular assays, we find that TAOK2 directly binds microtubules with high affinity. We define the minimal TAOK2 determinants that induce ER-microtubule tethering, and delineate the mechanism for its autoregulation. While ER membrane dynamics are increased in TAOK2 knockout cells, the movement of ER along growing microtubule plus-ends is disrupted. We show that ER-microtubule tethering is tightly regulated by catalytic activity of TAOK2 in both interphase and mitotic cells, perturbation of which leads to profound defects in ER morphology and cell division. Our study identifies TAOK2 as an ER-microtubule tether, and reveals a kinase-regulated 


\section{INTRODUCTION}

28 The endoplasmic reticulum is an expansive and complex membranous cellular

29 organelle. Composed of a continuous interconnected web of membrane sheets and

30 tubules, the ER has distinct domains; namely the nuclear envelope, the rough ER sheets

31 and smooth peripheral ER tubules (Voeltz et al., 2002). In addition, the ER makes

32 specialized membrane contact sites with other organelles and the plasma membrane

33 (Scorrano et al., 2019; Wu et al., 2018). Each of these domains are thought to be

34 structurally discrete regions of the ER serving specialized physiological functions. This

35 vast network of ER membranes relies on the microtubule cytoskeleton not only for

36 structurally supporting its intricate shape and functional domains, but also for its motility

37 and remodeling in response to stimuli. In animal cells, ER tubules align along

38 microtubules (Terasaki et al., 1986). Disruption of microtubules by depolymerization

39 agent nocodazole collapses the reticulated ER network into primarily ER sheets around

40 the nucleus (Terasaki and Reese, 1994). In addition to its structural dependence,

41 movement of ER membrane tubules occurs on microtubule tracks. Stabilization of

42 microtubules by the drug taxol prevents new microtubule growth, and also inhibits ER

43 tubule extension (Terasaki and Reese, 1994). Motor mediated ER 'sliding' movement

44 occurs on stable acetylated microtubules (Friedman et al., 2010). Motor independent ER

45 tubule extension along growing microtubule plus-ends is also dependent on

46 microtubules, and is carried out by the 'tip attachment complex' composed of ER protein

47 Stim1 and microtubule plus-end protein EB1 (Waterman-Storer and Salmon, 1998).

48 Additionally, membrane contact sites between ER and organelles appear to be

49 supported by microtubules. For example, ER-mitochondria contact sites are

50 preferentially aligned with acetylated microtubules (Friedman et al., 2010). Endosome

51 maturation occurs at junctions where ER-endosome contact sites and microtubules

52 converge (Wu and Voeltz, 2021). Store-operated calcium entry occurs through the 
53 Stim1-Orai channels at ER-plasma membrane contact sites (Grigoriev et al., 2008).

54 Stim1 interaction with microtubules plays a facilitative role in organizing Stim1 for optimal

55 Ca2+ sensing through its interaction with Orai channels (Smyth et al., 2007). In

56 geometrically complex cells such as neurons, the ER is dependent on microtubules for

57 its distribution throughout the fine processes such as dendrites, dendritic spines and

58 axons. Presence of fine caliber ER tubules in axons is critical for neuronal polarity, and

59 is dependent on ER membrane interactions with microtubules (Farías et al., 2019). On

60 the other hand, unlike elongated ER in axons and dendrites, which is closely associated

61 with microtubules, ER organization at dendritic branch points and dendritic spines is

62 structurally complex and exhibits decreased microtubule association (Cui-Wang et al.,

63 2012). Bidirectional regulation of ER-microtubule tethering, therefore not only influences

64 ER morphology but can also dictate cell shape and function.

Identity of molecular tethers that mediate ER-microtubule coupling, and mechanisms through which tethering is physiologically regulated are not well understood. Studying ER-microtubule association and its feedback control is challenging, owing to the considerably expansive morphology of the ER, one that exhibits extreme overlap with the microtubule cytoskeleton. The nanoscale organization of sites at which ER is coupled with microtubules and its molecular composition is

71 unclear. Constantly in flux, ER membranes undergo a range of dynamic and structural

72 changes including membrane extension, retraction, three-way junction formation and

73 tubule fusion (Pendin et al., 2011) . Cell division brings about extensive ER remodeling,

74 causing the ER membranes to coalesce around the spindle poles, but remain largely

75 absent from the mitotic microtubule spindle at metaphase (Jongsma et al., 2015);

76 (Smyth et al., 2015). Before insight into ER-microtubule dynamics and its physiological

77 regulation can be gained, the mechanisms through which molecular tethers drive ER-

78 microtubule association must be understood. Here, we investigate ER-microtubule 
79 association in interphase and mitotic human cells, and identify TAOK2 as an ER-

80 localized multifunctional protein kinase that serves as a molecular tether linking ER to

81 microtubules. An ER-microtubule tether should in principle be ER localized and be

82 capable of direct and strong binding to microtubules. The tether would likely be under

83 physiological regulation such that ER-microtubule binding can not only be ectopically

84 induced, but also importantly turned off. Loss of molecular tether should disrupt ER

85 association with microtubules. Our study demonstrates that the kinase TAOK2 meets all

86 the above criteria of a bona fide ER-microtubule tethering molecule.

87 Thousand And One amino acid (TAO) kinases are ubiquitously expressed

88 serine/threonine protein kinases belonging to the Ste20 kinase family (Chen et al., 1999;

89 Manning et al., 2002). While there is only one Tao kinase encoding gene in

90 invertebrates, three distinct TAOK genes are expressed in human (Chen et al., 2003;

91 Manning et al., 2002). The encoded kinases TAOK1, TAOK2 and TAOK3 share a highly

92 conserved N-terminal kinase domain, followed by distinct C-terminal domains (Chen et

93 al., 2003; Manning et al., 2002). TAO kinases were originally identified as stress-

94 sensitive kinases that activate the p38 kinase cascades through activation of MEK

95 kinases (Chen et al., 1999). Among the TAO family of protein kinases, TAOK2

96 coordinates several aspects of neuronal development and function. TAOK2 is highly

97 expressed during neuronal development and is important for basal dendrite formation as

98 well as for axon elongation in cortical neurons (de Anda et al., 2012). TAOK2 is

99 enriched in dendritic spines and is essential for their formation and stability (de Anda et

100 al., 2012; Ultanir et al., 2014; Yadav et al., 2017). TAOK2 knockout mice exhibit

101 cognitive and social-behavioral deficits, and show structural changes in brain size

102 (Richter et al., 2018). Recently, mutations is TAOK2 have been associated with autism

103 spectrum disorder, with several mutations present outside the kinase domain of the

104 protein (Richter et al., 2018). Despite its clear relevance to human development and its 
105 disease association, little is known about the molecular and cellular functions of TAOK2

106 kinase.

107 In our study, we demonstrate that TAOK2 is a pleiotropic protein, with distinct

108 catalytic kinase and ER-microtubule tethering functions, mediated through defined and

109 dissociable domains. TAOK2 is enriched at junctions where the ER membrane makes

110 contacts with the microtubule cytoskeleton. We show that TAOK2 is embedded in the

111 ER membrane through transmembrane helices, and an amphipathic region that limits its

112 localization to discrete subdomains of the ER. The cytoplasm facing ER-anchored C-

113 terminal tail of TAOK2 directly binds microtubules with high specificity, and is essential

114 for tethering of the ER membranes to microtubules. The ER and microtubule binding

115 domains together, a remarkably small region of 100 residues, are sufficient for inducing

116 ectopic tethering of ER to microtubules. Aberrant tethering by this minimal tether

117 abolishes ER dynamics and mitotic ER remodeling. Importantly, we show that the

118 tethering function of TAOK2 is negatively regulated by its kinase activity. During mitosis,

119 we find that TAOK2 is highly activated, and inhibition of its catalytic function prevents ER

120 disengagement from the mitotic spindle causing profound mitotic defects. This study

121 identifies TAOK2 as an ER protein kinase and elucidates a hitherto unknown

122 autoregulated mechanism for ER-microtubule tethering important for ER dynamics and

123 mitotic segregation.

\section{RESULTS}

126 TAOK2 is a multi-transmembrane protein kinase that resides on the endoplasmic

127 reticulum

128 To investigate the unique role that non-kinase domains of TAO family members might

129 impart on their biological function, we performed bioinformatic analysis of secondary

130 protein structure of TAO kinases. We found that TAOK2 $\alpha$ (hereafter TAOK2) harbors 
131 unique hydrophobic regions in its C-terminal domain not present is its alternatively

132 spliced isoform TAOK2 $\beta$ or paralogous genes TAOK1 and TAOK3. Sequence analysis

133 of TAOK2 through the transmembrane helix prediction software TMHMM2.0 (Krogh et

134 al., 2001) indicates that TAOK2 is a multipass membrane protein containing four

135 transmembrane helices (Figures $1 \mathrm{~A}$ and $1 \mathrm{~B}$ ). Additional predicted region of

136 hydrophobicity following the fourth transmembrane domain was analyzed using

137 AMPHIPASEEK (Combet et al., 2000) and HeliQuest (Gautier et al., 2008). This analysis

138 revealed an amphipathic helical $(\mathrm{AH})$ region with a sharply defined hydrophobic face

139 (hydrophobicity $<\mathrm{H}>=0.75$, hydrophobic moment $<\mathrm{mM}>=0.4$ ) and a polar face rich in

140 positively charged residues (net charge $z=4)$ (Figure $1 \mathrm{~A}$ inset).

141 To determine the cellular localization of the membrane spanning TAOK2 kinase,

142 we generated a rabbit polyclonal antibody against the unique C-terminal tail (residues

143 1220-1235) of TAOK2 $\alpha$ (Figure 1B). Antibody staining in HEK293T cells revealed that

144 endogenous TAOK2 colocalized extensively with the ER protein calreticulin, as well as

145 with the expressed ER marker EGFP-Sec22b (Figures S1A and S1B). We noted that

146 TAOK2 exhibited a striking localization on subdomains of the ER membrane in distinct

147 punctate pattern. Therefore, we generated a GFP-tagged TAOK2 construct and

148 performed super resolution microscopy (super-resolution by optical reassignment using

149 SoRa disk) to visualize the ER localization of TAOK2 at higher resolution (Figure 1C).

150 GFP-TAOK2 localized on the ER membrane and was present in discrete membrane

151 subdomains (Supplementary Movie 1). To test biochemically whether TAOK2 is an ER

152 membrane protein, we fractionated HEK293T cell homogenates into ER membrane

153 fraction using differential centrifugation (Hoyer et al., 2018). TAOK2 was enriched in the

154 ER membrane fraction along with other known ER membrane proteins such as Stim1

155 (Grigoriev et al., 2008) and Rtn3a (Hu et al., 2009). 97.6\% of the total TAOK2 in the 
156 postnuclear homogenate was enriched in the ER fraction, as compared to $6.17 \%$ of

157 tubulin (Figure 1D). On differential centrifugation, TAOK2 partitioned in the membrane

158 fraction but not the cytoplasmic fraction, and treatment with detergent led to its release in

159 the supernatant (Figure 1E). To identify the mechanism through which TAOK2 achieves

160 its ER localization, we generated four GFP-tagged deletion constructs (Figure 1F).

161 Deletion construct lacking the transmembrane and amphipathic helices (1-622) was

162 entirely cytosolic, while the four transmembrane helices alone (941-1066) were sufficient

163 to target the kinase to the ER. A GFP-construct containing just the TAOK2

164 transmembrane domains localized uniformly throughout the ER, and was not restricted

165 to ER subdomains. We found that the predicted amphipathic helical region together with

166 the transmembrane domains (941-1162) is required for localization of TAOK2 to discrete

167 ER subdomains, and captures the localization patters of the endogenous TAOK2 protein

168 (Figure 1F). Therefore, using superresolution microcopy and biochemical assays, we

169 show that TAOK2 is an ER resident protein kinase. Further, our data show that TAOK2

170 associates with the ER through its four transmembrane, while the amphipathic helices

171 confer its localization to distinct punctate ER subdomains.

173 TAOK2 associates directly with assembled microtubules via its conserved C-

\section{4 terminal tail}

175 TAOK2 has previously been shown to colocalize with microtubules (Mitsopoulos et al.,

176 2003), however mechanism underlying this observation is unknown. We found that

177 overexpression of the C-terminal TAOK2 constructs led to bundling of microtubules, and

178 these bundles were extensively decorated with GFP-TAOK2 constructs (Figure 2A and

179 2B). Expression of C-terminal GFP-tagged deletion constructs allowed us to map the

180 microtubule-binding domain to 40 amino acids (1196-1235) in the extreme C-terminal tail

181 of TAOK2 (Figure 2C). We next tested whether TAOK2 can bind microtubules directly 
182 using a biochemical binding assay with purified components. We bacterially purified GST

183 tagged TAOK2 C-terminal tail protein (residues 1187-1235) (Figure S2A). An in vitro

184 microtubule binding assay was used to test whether purified GST-TAOK2-C could bind

185 microtubules polymerized from purified tubulin protein. GST-TAOK2-C pelleted

186 specifically with polymerized MTs on centrifugation, while the control GST protein

187 remained in the supernatant (Figure 2D and 2E), suggesting TAOK2 can directly

188 associate with microtubules via its C-terminal tail. Further, the affinity of the TAOK2-

189 microtubules interaction was assessed by determining the fraction of microtubule bound

190 TAOK2-C at increasing concentration of tubulin. We found that the $C$ terminal tail of

191 TAOK2 associates with microtubules with a $\mathrm{K}_{\mathrm{D}}$ of $0.67 \pm 0.19 \mu \mathrm{M}$ (Figure $2 \mathrm{~F}$ and $2 \mathrm{G}$ ).

192 These results show that TAOK2 can directly bind microtubules through its cytoplasm

193 facing C-terminal tail.

195 ER membrane tethering to the microtubule cytoskeleton is mediated by TAOK2

196 Our finding that TAOK2 is an ER kinase with the ability to directly bind microtubules led

197 us to hypothesize that this enzyme can act as a molecular tether linking the ER

198 membrane to microtubules (Figure 3A). To evaluate this possibility, we performed live

199 cell imaging with simultaneous visualization of the ER membrane and microtubule

200 cytoskeleton. Cells transfected with full length GFP-TAOK2 and ER-mRFP were

201 incubated with a cell permeable microtubule binding dye, and three color time-lapse

202 confocal microscopy was performed (Supplementary Movie 2). Indeed, 87\% of GFP-

203 TAOK2 punctae localized at the points of contact between the ER and the microtubule

204 cytoskeleton (Figure 3A-3C). These punctae tracked with the movement of the ER

205 membrane on microtubules (Supplementary Movie 2). In order to ascertain the domains

206 within TAOK2 that conferred the tethering function of TAOK2, we generated several

207 constructs that retained the ER localization and microtubule binding elements. The first 
208 included the transmembrane domain (TMD), the amphipathic helix (AH) and microtubule

209 binding domain, but lacked the N-terminal kinase and coiled coil domains (Figure 3D, top

210 row). We found that expression of this construct led to aberrant over-tethering perturbing

211 ER morphology, such that the ER appeared bundled alongside perinuclear microtubule

212 cables. Next, we expressed a minimal-tether construct containing just the $\mathrm{AH}$ linked to

213 the microtubule binding domain (1146-1235). Expression of the 'mini-tether' led to a

214 complete collapse of the ER on to the microtubule cytoskeleton, where the ER

215 membrane was entirely conformed to the shape of the microtubule cytoskeleton (Figure

$2163 \mathrm{D}$, center row). Live imaging revealed that aberrant tethering induced by the minimal-

217 tether abolishes ER membrane dynamics due to its collapse on microtubules

218 (Supplementary Movie 3). As anticipated, the TMD and the AH domain without the

219 microtubule binding domain failed to induce tethering, and the ER retained its lace-like

220 morphology occupying the entire cell area (Figure 3D, bottom row). These data together

221 define the mechanisms through which TAOK2 mediates its tethering function, and

222 delineate the minimal elements comprising the ER-microtubule tether. Importantly, these

223 findings also imply that additional domains of TAOK2 outside the tethering elements

224 regulate the coupling dynamics of the ER to the microtubule cytoskeleton.

226 TAOK2 is required for ER microtubule plus end motility but not motor mediated

\section{7 movement}

228 The ER membranes utilize the microtubule cytoskeleton as tracks for

229 movement ${ }^{13-15}$. Distinct mechanisms of ER motility have been defined; 'sliding'

230 movements are kinesin-based rapid movement, and 'tip-attachment complex'

231 movements occur when the ER attaches to the MT plus ends and tracks along the

232 growing MT (Friedman et al., 2010; Waterman-Storer and Salmon, 1998). To determine

233 the functional consequences of TAOK2 depletion on ER motility and ER-MT movement, 
234 we generated two independent TAOK2 knockout (KO) HEK293T cell lines using

235 CRISPR/Cas9 mediated gene editing. Genetic knockout and loss of protein was

236 validated using genome sequencing and western blot respectively (Figure 4A, Figure

237 S2B and S2C). Wildtype and TAOK2 KO cells were transfected with the ER marker

238 EGFP-Sec22b and time-lapse confocal microscopy allowed us to visualize ER

239 dynamics. To test whether loss of TAOK2 mediated tethering would impact overall

240 motility of the ER membranes, we measured ER membrane movement over time. ER

241 motility in TAOK2 KO cells was significantly increased compared to WT cells (Figure

242 4B). Average normalized pixel differences over time allowed us to calculate the ER

243 motility index which increased from $0.287 \pm 0.012$ in WT cells to $0.351 \pm 0.017(n=9$,

$244 \mathrm{p}=0.0098$ ) in TAOK2 KO cells (Figure 4C). To further investigate how absence of TAOK2

245 mediated tethering might increase ER motility, we performed simultaneous confocal

246 imaging of ER (EGFP-Sec22b) and microtubule end binding protein mCherry-EB3. We

247 found that in WT cells both TAC movements on microtubule plus end, as well as sliding

248 ER movements were observed, however, in TAOK2 KO cells almost all observed

249 movements were mediated by sliding movements. Thus, loss of TAOK2 disrupts TAC

250 movements (Figure 4D), while motor mediated ER sliding movements are maintained

251 (Figure 4E). Next, we determined whether absence of TAOK2 would impact microtubule

252 dynamics. We found that microtubule growth assessed by measuring EB3 velocity was

253 slightly increased in TAOK2 KO cells (Figure 4F). Further, in TAOK2 knockout cells, EB3

254 comet tracks were less directed exhibiting increased curvature and paused more

255 frequently compared to control WT cells (Figure 4F-4G, Supplementary Movie 4). Thus,

256 our analyses of the TAOK2 knockout reveal an important role of TAOK2 in regulating the

257 dynamics of ER-microtubule based movement, and suggest that TAOK2 mediated ER-

258 MT tethering is essential for the structure and dynamics of ER membranes. 


\section{Aberrant TAOK2 tethering disrupts ER restructuring during mitosis}

261 The endoplasmic reticulum undergoes dynamic restructuring during cell division

262 (Carlton et al., 2020). During metaphase, as the mitotic spindle aligns the chromosomes

263 at the metaphase plate, the ER is anchored at each end to the spindle poles but largely

264 absent from chromosomes and the area between the spindle poles. Defects in ER

265 clearance from the chromosomes and spindle elicit mitotic defects (Schlaitz et al., 2013).

266 Given the extensive association of ER with MT during cell division, we investigated the

267 role of TAOK2 in ER restructuring during mitosis. Immunofluorescence using the C-term

268 TAOK2 antibody revealed that TAOK2 was present as discrete puncta throughout the

269 ER, the spindle pole, and on the spindle MT during mitosis (Figure 5A). We performed

270 superresolution confocal microscopy to visualize GFP-TAOK2 localization in mitotic

271 cells. We found that GFP-TAOK2 was present in close apposition to the spindle poles at

272 sites where the ER membranes converged. TAOK2 localized at discrete punctate sites

273 throughout the curvilinear peripheral ER surrounding the mitotic cell, as well as at the

274 points of contact between ER membrane and the mitotic spindle (Figure 5B, bottom

275 row). To test, whether TAOK2 was important for ER structural remodeling during cell

276 division, we imaged WT and TAOK2-KO HEK293T mitotic cells expressing EGFP-

277 Sec22b and mCherry-EB3 to visualize ER membranes and mitotic spindle. WT cells

278 exhibited the characteristic ER morphology showing accumulation at spindle poles and

279 fenestrated curvilinear peripheral ER. In contrast, TAOK2-KO cells had an abnormal

280 morphology with increased peripheral curvilinear ER membranes, and concomitant

281 decrease in association with spindle poles (Figure 5C and 5D). To determine if this

282 aberrant ER morphology affected cell division, we immunostained WT and TAOK2 KO

283 cells with tubulin and DAPI. While 95.6\% WT cells showed normal bipolar spindle, only

$28444.8 \% \mathrm{KO}$ cells had a normal bipolar spindle. KO mitotic cells displayed a chromosomal

285 misalignment defect, where $39.3 \% \mathrm{KO}$ cells had a bipolar spindle with misaligned 
286 chromosomes, and $15.8 \% \mathrm{KO}$ cells had multipolar spindle (Figure 5E). While loss of

287 TAOK2 induced defects in ER association with mitotic spindle, we next queried what

288 would be the consequence of unregulated over-tethering by TAOK2. Expression of the

289 short TAOK2 lacking the N-terminal kinase and coiled coil domains, induced a dramatic

290 collapse of the ER membranes on the mitotic spindle (Figure 5F). TAOK2 binding to

291 microtubules created an extremely short and stable MT-ER bridge between the spindle

292 poles, which we refer to as pseudomonopolar (90.5\% mitotic cells). The chromosomes

293 were displaced from the metaphase plate and instead formed a rosette around the ER-

294 MT spindle (Figure 5F, top row). These cells failed to divide. Expression of the 'mini-

295 tether' construct containing the $\mathrm{AH}$ and the microtubule binding domain, generated a

296 similar phenotype of pseudomonopolar spindle (30.7\% mitotic cells) where the ER

297 remained attached to the microtubules. Additionally, cells expressing the minimal tether

298 displayed aberrant spindles which did not appear to be focused on the spindle poles

299 (61.5\% mitotic cells). In all the cases, the chromosomes were displaced from the spindle

300 and ER was tightly associated with the spindle microtubules. The number of normal

301 bipolar cells decreased from $96.7 \%$ in cells expressing WT-TAOK2 to $1.96 \%$ and $3.93 \%$

302 in mitotic cells expressing the tether constructs TAOK2 (941-1235) and TAOK2 (1146-

303 1235), respectively. However, expression of a TAOK2 construct lacking the microtubule

304 binding domain TAOK2 (941-1162), had normal ER morphology during mitosis, and

$30592.2 \%$ mitotic cells had a bipolar normal spindle (Figure 5F and 5G). These data

306 collectively show that downregulation of TAOK2 mediated ER-MT tethering during cell

307 division is required for the disengagement of ER from the mitotic spindle and its

308 segregation into daughter cells.

309

310 Catalytic autoregulation of TAOK2 mediated ER-MT coupling 
What might be the mechanism through which TAOK2 regulates its function as an

312 ER-MT tether? We tested whether the kinase activity of TAOK2 regulates its microtubule

313 association. First, we introduced a kinase-dead mutation within the catalytic domain of

314 TAOK2 at residue K57A, which disrupts the autophosphorylation at the critical residue

315 S181 in the activation loop (Moore et al., 2000), and hence the catalytic activity of

316 TAOK2 (Figure 6A). Comparative analysis of cells expressing ER-mRFP along with

317 either GFP tagged-TAOK2 WT or TAOK2-K57A revealed that loss of kinase activity

318 increased the association of TAOK2 with microtubules (Figure 6B). Colocalization

319 analysis of GFP positive TAOK2 puncta with tubulin showed that association with

320 microtubules increased from $87.86 \pm 2.17 \%$ for TAOK2-WT to $96.46 \pm 1.28 \%$ for TAOK2-

321 K57A (Figure 6C). To test if increased association with microtubules resulted in stronger

322 ER-MT tethering, we measured ER motility in cells expressing kinase dead TAOK2-

323 K57A and ER marker EGFP-Sec22b. A substantial decrease in ER membrane motility

324 was found in cells expressing TAOK2-K57A as opposed to TAOK2-WT (Figure 6D and

325 Supplementary Movies 5-6). The ER motility index calculated from mean normalized

326 pixel differences over time, was found to decrease from $0.28 \pm 0.006$ in TAOK2-WT to

$3270.23 \pm 0.01(n=10, p=0.0029)$ in K57-TAOK2 expressing cells (Figure 6E). Further, we

328 found that TAOK2 kinase activity was important for microtubule growth rates determined

329 by measuring EB3 comet velocity. EB3 velocity averaged at $0.35 \mu \mathrm{m} / \mathrm{s}$ in TAOK2-WT

330 expressing cells and was decreased significantly to $0.17 \mu \mathrm{m} / \mathrm{s}$ in cells expressing kinase

331 dead TAOK2 (Figure 6F and Supplementary movies 5-6). While directional persistence

332 of MT growth was reduced, the frequency of MT comet pauses increased from $8.5 \%$ in

333 control to $19.7 \%$ in K57A-TAOK2 expressing cells (Figure S3A and S3B). These data

334 indicate that catalytic activity of TAOK2 regulates its ER-MT tethering function. While 
335 TAOK2 kinase activity negatively regulates its association with microtubules, it positively

336 regulates microtubule growth and dynamics.

337 Next, we assessed whether TAOK2 catalytic activity was regulated during

338 mitosis, and found that TAOK2 was highly activated during mitosis. Immunostaining

339 interphase and mitotic cells with phospho-S181 antibody revealed a 2-fold increase in

340 mitotic cells compared to interphase (Figure 6G). Western blot analysis showed high

341 ratio of pS181-TAOK2/TAOK2 in lysates from synchronized mitotic cells compared to

342 asynchronous cell lysate (Figure $6 \mathrm{H}$ ). Therefore, we tested if perturbation of TAOK2

343 kinase activity would impact mitosis or ER segregation during cell cycle. We expressed

344 either GFP tagged TAOK2-WT or TAOK2-K57A along with ER-mRFP, and then used a

345 405nm-DNA dye and 673nm-MT dye to enable four-color imaging of TAOK2, ER

346 membrane, chromosomes and mitotic MT spindle. We found that while in TAOK2-WT

347 expressing cells, both TAOK2 and the ER membranes were enriched at the spindle

348 poles, and a majority of the ER membrane was dissociated from the spindle

349 microtubules. However, in cells expressing TAOK2-K57A kinase dead mutant, TAOK2

350 association with the spindle MT was markedly increased and ER membranes were

351 extensively associated with the mitotic spindle MT (Figure 6I). A failure of ER

352 membranes to disengage from the spindle MT led to severe mitotic defects including

353 monopolar (22\%), multipolar (15\%) and aberrant spindles (21\%). Accordingly, the

354 number of mitotic cells exhibiting a normal bipolar spindle deceased from $92 \%$ in cells

355 expressing TAOK2-WT to about 41\% in TAOK2-K57A expressing cells (Figure 6J).

356 These data demonstrate that the kinase activity of TAOK2 is important for dynamic

357 regulation of ER-MT tethering during cell division, and perturbation of its catalytic activity

358 leads to failure of ER segregation into daughter cells, ultimately inducing mitotic defects. 
Our study identifies TAOK2 as an ER resident kinase that functions as a

362 molecular tether linking the ER membranes to microtubule cytoskeleton. TAOK2 is a

363 large multifunctional protein of 1235 amino acids (Chen et al., 1999). At the molecular

364 level, we show that distinct structural domains within TAOK2 confer its catalytic activity,

365 ER localization and microtubule association. Importantly, we demonstrate that, while the

366 ER-microtubule tethering function of TAOK2 is structurally dissociated from its kinase

367 domain, the catalytic activity of TAOK2 negatively regulates ER-microtubule tethering.

368 Our results show that knockout of TAOK2 and kinase-dead TAOK2 expression have

369 opposing effects on ER-microtubule tethering and dynamics in both interphase and

370 mitotic cells. Thus, TAOK2 possesses the unique capacity to autoregulate ER-

371 microtubule tethering through its kinase activity. We provide two key pieces of evidence

372 in support of bidirectional autoregulation of tethering by TAOK2. First, we show that the

373 kinase dead TAOK2 is a stronger ER-microtubule tether. Second, TAOK2 kinase activity

374 is increased in mitosis, which correlates with a dramatic decrease in the tethering of ER

375 membranes with the mitotic spindle microtubules. Perturbation of TAOK2 kinase activity

376 in mitotic cells leads to failure in disengagement of ER from the spindle causing mitotic

377 defects.

Little is known about upstream mechanisms that mediate TAOK2 activation.

380 Early studies on the family of TAO kinases suggest that they might function as

381 intermediate signaling kinases that link certain heterotrimeric G protein-coupled

382 receptors to the p38 MAPK pathway. Among ligands that induce TAOK2 activation,

383 nocodazole, sorbitol and the muscarinic agonist carbachol were found to increase

384 TAOK2 activity from 1.5-3 fold on stimulation (Chen et al., 2003). Members of the TAO

385 family can be activated by ATM kinase is response to genotoxic stress (Raman et al.,

386 2007), however, whether TAOK2 is phosphorylated is unknown. TAOK2 is 
387 phosphorylated by the Hippo kinase mammalian homolog MST3 at residue T468

388 proximal to the kinase domain (Ultanir et al., 2014). Whether phosphorylation by MST3

389 increases TAOK2 catalytic activity has not been demonstrated. Further, in neurons, the

390 secreted semaphorin molecule Sema3a has been shown to increase TAOK2 kinase

391 activity in neurons through interaction with its receptor Neuropilin 1 (de Anda et al.,

392 2012). Therefore, several independent signaling pathways might impinge on TAOK2

393 kinase to achieve distinct context-dependent cellular outcomes.

Our findings show that TAOK2 is a unique serine/threonine kinase, as no other

396 multipass membrane serine/threonine kinase has been reported to date. Two other

397 kinases with a single transmembrane domain reside in the ER membrane, Ire1(Cox et

398 al., 1993; Mori et al., 1993) and PERK (Harding et al., 1999). Both Ire1 and PERK

399 kinases have a cytosol facing kinase domain similar to TAOK2. The C-terminal tails of

400 Ire1 and PERK project into the luminal domain where they have important functions

401 associated with sensing ER stress (Ron and Walter, 2007). In contrast, the C-terminal

402 tail of TAOK2 faces the cytoplasm and directly binds microtubules. The intrahelical loops

403 between with the transmembrane helices are predicted to be extremely short (4-6 amino

404 acids), and it is unlikely that TAOK2 serves a role as an ER stress sensor within the ER

405 lumen. However, it is conceivable that TAOK2 is activated during ER stress indirectly

406 through other ER stress sensors. Our data suggests that the punctate localization of

407 TAOK2 within distinct ER subdomains is conferred by an amphipathic helical domain

408 following the fourth transmembrane helix. Investigating the molecular identity of these

409 ER subdomains in terms of its lipid and protein constituents would likely reveal important

410 insight into TAOK2 regulation. Based on the findings of this study, we predict that

411 increased activation in conditions of cellular stress might negatively regulate its function

412 as an ER-MT tether, and will be an important area of future inquiry. 
414 tether, adds to the short list of two other multifunctional ER enzymes, Spastin and

415 Atlastin that bind microtubules. Hereditary spastic paraplegia protein, Spastin, is a

416 microtubule severing AAA ATPase enzyme that localizes to the ER and remodels

417 microtubules via its C terminus domain (Hazan et al., 1999; Roll-Mecak and Vale, 2008).

418 The other is Atlastin, an ER localized GTPase required for ER tubule fusion (Orso et al.,

419 2009). In addition to these enzymes, several other ER proteins associate with the

420 microtubule cytoskeleton directly or indirectly, and are each likely to serve a particular

421 physiological function through tethering (Wang et al., 2016). Perhaps the most well

422 studied, Stim1, is an ER protein that interacts with microtubule growing tips indirectly by

423 binding EB1 proteins (Grigoriev et al., 2008; Pavez et al., 2019). Climp63 is an ER

424 protein thought to instruct the luminal width of ER sheets (Nikonov et al., 2007;

425 Vedrenne et al., 2005) and can directly associate with microtubules (Klopfenstein et al.,

426 1998). It is important to note, that unlike the abovementioned microtubule binding ER

427 proteins, TAOK2 has the unique ability to bidirectionally autoregulate its microtubule 428 association.

As a kinase highly expressed in neurons, and one critical for neurodevelopment,

431 the role of TAOK2 as an autoregulated ER-MT tether is likely to serve specific

432 physiological functions in neurons. Neuronal development, connectivity and plasticity are

433 dependent on the presence of ER membranes within fine neuronal processes such as

434 spines, axons and dendrites where they are transported along and tethered to

435 microtubules. TAOK2 is critical for dendritic spine development (Ultanir et al., 2014;

436 Yadav et al., 2017), axon elongation and basal dendrite branching (de Anda et al., 2012;

437 Richter et al., 2018). TAOK2 knockout mouse models exhibit cognitive and social-

438 behavioral deficits, and show structural changes in brain size (Richter et al., 2018), 
439 mechanisms of which are unknown. Further, de novo mutations in TAOK2 have been

440 found through whole-genome and exome sequencing of patients with autism spectrum

441 disorder (Richter et al., 2018). Our findings provide a hitherto unknown mechanism for

442 kinase mediated control of ER tethering to microtubule cytoskeleton. Elucidating TAOK2

443 function in specialized cells types such as neurons that respond to physiological stimuli

444 by remodeling ER-microtubules tethering is likely to expand on our current

445 understanding of the importance and dynamics of communication between cellular

446 organelles and the cytoskeleton.

\section{Acknowledgement}

449 We are grateful for research funding provided by National Institute of Mental Health, R00

$450 \mathrm{MH} 108648$ and R01 MH121674 to SY, the Brain and Behavior Research Foundation's

451 NARSAD Young Investigator Award (27818) to SY. KN was supported by the NIH pre-

452 doctoral Pharmacological Sciences Training Program T32GM007750. We thank Dan

453 Fong (Nikon) for technical assistance on the Nikon CSU-W1 SoRa superresolution

454 microscope. We are grateful to Brian Beliveau (UW), Cole Trapnell (UW) and Jay

455 Shendure (UW, HHMI) for use of their Nikon CSU-W1 SoRa superresolution microscope

456 funded by HHMI. Thanks to John D. Scott (UW), Ning Zheng (UW, HHMI) and Shao-En

457 Ong (UW) for comments and suggestions on the manuscript.

\section{Author contribution}

460 All experiments were designed, performed and analyzed by $\mathrm{KN}$ and SY, unless

461 otherwise stated. AF generated and characterized the TAOK2 KO lines and cloned the

462 GFP-TAOK2 construct. MB performed molecular biology experiments. Project funding

463 obtained by SY. Manuscript written by KN and SY, and edited by all authors. 


\section{Figure Legends}

Figure 1. TAOK2 is an ER protein kinase that localizes to the ER membrane

467 through four transmembrane domains and an amphipathic helix

468 (A) Transmembrane Hidden Markov Model (TMHMM v2.0) prediction plot shows the

469 posterior probabilities (y axis) of inside/outside(magenta) / TM helix (red) along the

470 length of TAOK2 sequence (x axis). Hydrophobic region following the $4^{\text {th }}$ predicted TMD

471 was analyzed through AMPHIPASEEK which predicted residues 1146-1162 to be

472 amphipathic. Charged (blue) and hydrophobic (yellow) residues are indicated in the

473 inset. HeliQuest was used to calculate hydrophobicity $<\mathrm{H}>$, hydrophobic moment $<\mu \mathrm{H}>$

474 and net charge $z$.

475 (B) Schematic representation of TAOK2 isoforms $\alpha, \beta$ and TAOK1. The coiled coils

476 (CC), four transmembrane domains (TM) and amphipathic helix (AH) predicted in

477 TAOK2 are depicted. The unique C-terminal tail region of TAOK2 marked by the asterisk

478 indicates the epitope used to generate the TAOK2 $\alpha$ specific antibody.

479 (C) Super resolution (SoRa: super resolution by optical reassignment) images of

480 HEK293T cells expressing GFP-TAOK2 (cyan) and ER marker ER-mRFP (red), zoomed

481 in sections are depicted in yellow boxes. Scale bar bottom left is $5 \mu \mathrm{m}$, and bottom right

482 is $0.5 \mu \mathrm{m}$.

483 (D) Western blot of cell homogenate fractionated into ER membrane and cytosol probed

484 with antibodies against TAOK2, known ER membrane proteins Stim1 and Rtn3,

485 mitochondrial protein Tom20 and tubulin.

486 (E) Western blot of cell homogenate fractionated into membrane pellet (P) and cytosolic

487 supernatant (S) components, in the absence (control) or presence of detergent, probed

488 with antibodies against TAOK2 and ER protein Rtn3a. 
489 (F) Schematic shows the GFP-tagged TAOK2 deletion constructs used in Fig.1g.

490 (G) Confocal images of HEK293T cells expressing distinct GFP-tagged TAOK2 deletion

491 constructs (green) and ER-mRFP (magenta). Scale bar is $3 \mu \mathrm{m}$.

493 Figure 2. Direct binding of TAOK2 to microtubules through its C-terminal tail

494 (A) Confocal images of HEK293T cells expressing the indicated GFP-tagged TAOK2

495 construct immunostained for $\alpha$-tubulin. Scale bar is $3 \mu \mathrm{m}$.

496 (B) Zoomed in images showing the overlap of GFP-tagged TAOK2 with microtubules

497 immunostained for $\alpha$-tubulin. Scale bar is $1 \mu \mathrm{m}$. RGB profile of fluorescence intensity

498 peaks of TAOK2 (green) and a tubulin (red).

499 (C) Schematic representation of GFP-tagged deletion constructs used to map the

500 microtubule binding domain. Coiled coil (CC), transmembrane (TMD); amphipathic helix

$501(\mathrm{AH})$ domains are marked. Observed microtubule localization is indicated accordingly in

502 the checkbox.

503 (D) Coomassie stained SDS-page gel shows co-sedimentation of indicated proteins with

504 polymerized tubulin. Microtubule binding assay was performed with $5 \mu \mathrm{g}$ of GST or GST-

505 TAOK2-C (1187-1235) protein in the presence of taxol polymerized microtubules.

506 Binding is assessed by the fraction of protein pelleted with microtubules $(P)$ while

507 unbound protein is in supernatant (S).

508 (E) Percent protein bound to microtubules is plotted for each protein as indicated. Error

509 bars indicate standard error of mean, $n=3, p<0.0001$, one-way ANOVA.

510 (F) Coomassie stained SDS-page gel shows the amount of purified GST-TAOK2-(1187-

511 1235) unbound (S) or co-sedimented with polymerized microtubules (P) with increasing

512 concentrations of tubulin as indicated. 
513 (G) Strength of microtubule association was determined from dissociation constant $\mathrm{K}_{\mathrm{D}}$

514 derived by Michaelis-Menten equation. Error bars indicate standard error of mean, $\mathrm{n}=3$

515 replicates.

516

517 Figure 3. TAOK2 is an ER-microtubule tethering protein kinase

518 (A) TAOK2 (green) schematically depicted as a multipass transmembrane protein on the

519 ER (grey) has an N-terminal cytoplasm facing kinase domain and a C-terminal tail that

520 directly binds microtubules (magenta). Confocal images of HEK293T cells expressing

521 GFP-TAOK2 (yellow), ER-mRFP (magenta) and live-stained with microtubule dye

522 (cyan). Scale bar is $3 \mu \mathrm{m}$.

523 (B) Montage of confocal time lapse images shows TAOK2 (yellow) punctae colocalized

524 with both ER membrane (magenta) and microtubules (cyan). Scale bar is $1 \mu \mathrm{m}$.

525 (C) Percent TAOK2 puncta colocalized with ER and microtubules is plotted. Values

526 indicate mean, $\mathrm{n}=10$ cells and error bars indicate S.E.M.

527 (D) Schematic on the left depicts topology of TAOK2 deletion constructs corresponding

528 to representative confocal images of HEK293T cells expressing the indicated TAOK2

529 construct (yellow), ER-mRFP (magenta) and microtubules (cyan). Scale bar is $5 \mu \mathrm{m}$.

531 Figure 4. TAOK2 knockout disrupts ER-microtubule dynamics

532 (A) Western blot of lysate from wildtype and TAOK2 (knock out) KO cells generated

533 using CRISPR/Cas9 mediated gene editing. Guide RNA sequences used for the

534 knockout are shown.

535 (B) Confocal images of peripheral ER in wildtype and TAOK2 KO cells expressing ER

536 marker EGFP-Sec22b at a single time point (left column). Sum slice projection of

537 cumulative pixel difference in successive frames over 30s time period (right), where 
538 images were acquired every 3sec. Fluorescence intensity is pseudocolor coded, red

539 regions representing increased ER motility. Scale bar is $3 \mu \mathrm{m}$.

540 (C) ER motility index calculated by averaging the normalized pixel difference between

541 successive time frame over 30 s is plotted for wildtype and TAOK2 KO cells. Error bars

542 indicate S.E.M, n=9 cells, t-test.

543 (D) Montage of images acquired every 2s, of wildtype and TAOK2 KO cells expressing

544 EGFP-Sec22b and mCherry-EB3 shows movement of ER membranes (cyan) associated

545 with microtubule plus tips (red) in wildtype but not KO cells.

546 (E) Time lapse confocal images of cells expressing EGFP-Sec22b and mCherry-EB3

547 were analyzed and ER membrane movements classified into slow MT plus tip

548 associated-TAC (colocalized with EB3 comet) and fast motor driven sliding movements

549 (lacking association with EB3 comets). Percent MT plus tip associated (TAC) events and

550 sliding events is shown for WT and TAOK2 KO cells. Mean values are plotted, $\mathrm{n}=5$ cells

551 for each condition.

552 (F) Microtubule directional persistence, calculated as a fraction of perpendicular distance

553 between start/end points and the length of the actual path taken is plotted for wildtype

554 and TAOK2 knockout cells. Values indicate mean \pm S.E.M., $n=9$ cells with at least 5

555 comet paths measured per cell, two tailed t-test.

556 (G) Percent total time spent by EB3 comet pausing (no growth) is plotted for wildtype

557 and TAOK2 KO cells. Values indicate mean \pm S.E.M., $\mathrm{n}=9$ cells with at least 5 comet

558 paths measured per cell, two tailed t-test.

$559(\mathrm{H})$ EB3 comet tracks generated using the Manual tracking function in Fiji were used to

560 measure EB3 comet velocity by dividing the total distance traveled over time. Mean

561 values for wildtype and TAOK2 KO cells are plotted, error bars indicate S.E.M, n=9 cells

562 with 5 comets per cell, two tailed t-test. 


\section{Figure 5. TAOK2 localizes to the mitotic spindle and regulates mitotic ER}

\section{5 remodeling}

566 (A) TAOK2 antibody staining shows localization of endogenous TAOK2 (yellow) on the

567 ER (cyan) and mitotic spindle microtubules (red) in mitotic cells.

568 (B) Superresolution confocal image of mitotic cell expressing GFP-TAOK2 (yellow) and

569 ER-mRFP (blue) and stained with microtubule dye (magenta), scale bar is $2 \mu \mathrm{m}$ (top

570 right). Magnified view of the ER-MT tethering sites on the spindle is shown in the bottom

571 row, scale bar is $0.5 \mu \mathrm{m}$.

572 (C) Confocal live cell images of WT and TAOK2 KO mitotic cells expressing EGFP-

573 Sec22b (green) and mCherry-EB3 (magenta). Dashed white line drawn across the

574 spindle poles highlights the difference in spindle pole associated ER fraction in WT and

575 TAOK2 KO cells. Scale bar is $5 \mu \mathrm{m}$.

576 (D) ER distribution in mitotic cells is measured using Azimuthal Average of normalized

577 fluorescence intensity in each hemisphere demarcated by the mitotic spindle (where 0

578 and 180 correspond to the spindle poles) is plotted for WT (gray) and TAOK2 KO

579 (orange) cells. Average means are plotted, error bars are S.E.M., and n=3 cells.

580 (E) Percent mitotic WT (grey) and TAOK2 KO (orange) cells that exhibit bipolar,

581 multipolar or misaligned mitotic spindles are plotted. Values indicate mean \pm S.E.M.,

$582 \mathrm{n}>45$ mitotic cells from three different experiments, $\mathrm{p}<0.0001$ one-way ANOVA.

583 (F) Schematic (left) depicts topology of GFP-TAOK2 deletion constructs. Corresponding

584 confocal images of mitotic HEK293T cells expressing the indicated TAOK2 construct

585 (yellow), ER-mRFP (magenta), DNA (blue) and microtubules (cyan) are shown. Scale

586 bar is $5 \mu \mathrm{m}$. 
587 (G) Mitotic defects in cells expressing the indicated TAOK2 constructs is depicted as the 588 percent of mitotic cells exhibiting normal bipolar or aberrant spindles. Values indicate 589 mean \pm S.E.M., $\mathrm{n}=52$ cells from 3 different experiments.

591 Figure 6. ER-microtubule tethering is regulated by catalytic activity of TAOK2

592 (A) Catalytic activity of GFP-TAOK2 WT and GFP-TAOK2 K57A was measured in an in 593 vitro kinase reaction using autophosphorylation at S181 as the readout. Western blot

594 probed with phospho-S181 antibody to measure kinase activity and anti-GFP represent

595 the total amount of TAOK2.

596 (B) Confocal images of cells expressing GFP-TAOK2 WT and GFP-TAOK2 K57A

597 (yellow) along with ER-mRFP (blue) and live-stained with microtubule dye (red). Scale

598 bar is $5 \mu \mathrm{m}$.

599 (C) Percent of GFP-TAOK2 WT and GFP-TAOK2 K57A puncta colocalized with

600 microtubules is plotted. Values indicate mean, $n=10$ cells, error bars indicate S.E.M., $601 p<0.005$, two tailed t-test.

602 (D) Confocal images of peripheral ER in cells expressing TAOK2 WT or TAOK2 K57A

603 along with ER marker EGFP-Sec22b at a single time point (left column). Sum slice

604 projection of cumulative pixel difference in successive frames over 30s, where images

605 were acquired every 3s are shown. Fluorescence intensity is pseudocolor coded, red

606 regions representing increased ER motility. Scale bar is $3 \mu \mathrm{m}$.

607 (E) ER motility index is calculated by averaging the normalized pixel difference between

608 successive time frame over 30s. Mean ER motility for TAOK2 WT and TAOK2 K57A

609 expressing cells is plotted. Error bars indicate S.E.M, n=10 cells, two tailed t-test. 
610 (F) Montage of images acquired every 2s of cells expressing TAOK2-WT or TAOK2

611 K57A along with mCherry-EB3 shows movement of EB3 comets (cyan) over time on y

612 axis. Scale bar is $1 \mu \mathrm{m}$.

613 (G) EB3 comet tracks generated using the Manual Tracking function in Fiji were used to

614 measure EB3 comet velocity by dividing the total distance travelled over time. Mean

615 velocity values for cells expressing mCherry-EB3 along with either TAOK2 WT or

616 TAOK2 K57A are plotted. Error bars indicate S.E.M, n=10 cells, two tailed t-test.

617 (H) Confocal images of mitotic and interphase HEK293T cells stained with phospho-

618 S181 and tubulin antibodies to label active TAOK2 and microtubules respectively. DNA

619 stained with DAPI.

620 (I) Average intensity of phospho-S181 staining in mitotic cells normalized by average

621 interphase intensity is plotted. Error bars indicate S.E.M, n=10 cells, t-test with Welch

622 correction.

623 (J) Confocal images of mitotic cells expressing GFP-TAOK2 WT or GFP-TAOK2 K57A

624 (yellow) along with ER-mRFP (cyan) was stained with DNA dye (blue) and microtubules 625 dye (red). Scale bar is $5 \mu \mathrm{m}$.

626 (K) Mitotic defects in cells expressing GFP-TAOK2 WT or GFP-TAOK2 K57A are plotted

627 as the percent of mitotic cells exhibiting normal bipolar or aberrant spindles. Values

628 indicate mean \pm S.E.M., $n=50$ cells from 3 different experiments.

629 (L) Schematic representation of functional roles of TAOK2 in maintaining ER structure

630 and its remodeling during mitosis. Distinct effects of TAOK2 depletion (TAOK2 knockout)

631 compared to those due to kinase dysfunction (TAOK2 kinase dead) in interphase and

632 mitotic cells are shown. The divergent effects of TAOK2 kinase dead mutation and

633 TAOK2 knockout suggest pleiotropic roles of TAOK2 mediated by functional domains in

634 addition to its kinase domain. 


\section{Methods}

\section{Antibodies and Plasmids}

637 Antibodies used in this study are as follows: alpha-Tubulin (Mouse, Sigma, T9026-

638 100UL), GAPDH (Mouse, Invitrogen, MA5-151738), Calreticulin (Mouse, Abcam, 639 ab22683), TAOK2, Rabbit, Sigma, HPA010650), Rtn3a (Rabbit, ProteinTech,12055-2-

640 AP), Acetylated alpha Tubulin (Mouse, Sigma, T6793), GST (Mouse, Invitrogen),

641 GM130 (Mouse, BD Labs, 610822), TAOK2-Cterm (Rabbit), Tom20 (Mouse, Santa Cruz

642 Biotech, sc-17764), Stim1 (Mouse, Santa Cruz Biotech, sc-166840), GFP (Mouse,

643 Roche, 11814460 001), Phospho-TAO2 (S181) (Rabbit, R\&D Systems,

644 PPS037). Addgene Plasmids used in this study are as follows: mCh-Sec61 beta

645 (\#49155), sfGFP-C1 (\#54579), ER-mRFP (\#62236), EGFP-Sec22b (\#101918).

\section{Molecular Cloning and CRISPR/Cas9 genome editing}

647 Full length human TAOK2 was PCR amplified from pCMV-Sp6-TAOK2 plasmid

648 described previously (Ultanir et al., 2014), and inserted in vector sfGFP-C1 (Addgene

649 \#54579) using restriction sites HindlII and Mfel. Domain dissection mutants were

650 subcloned from sfGFP-TAOK2 using restriction enzymes Hindlll and Mfel (New England

651 Biolabs). All resultant plasmids were verified by sequencing. GST-TAOK2-(1187-1235)

652 was subcloned from the sfGFP-TAOK2 into the pGEX4T1 vector using sites Sall and

653 Notl. TAOK2 knockout cell line was generated using CRISPR/Cas9 genome editing in

654 HEK293T cells. Four independent guides were designed using Synthego guide design

655 tool (https://www.synthego.com) to target coding exon 2. Two plasmids were made by

656 adding their respective guides into CrisprV2pSpCas9(BB)-2A-Puro (PX459) V2.0

657 (Addgene Plasmid \#62988). Cells were passaged in single cell suspension and plated at

$65850 \%$ confluence. Cultures were then transfected with lipofectamine 2000 reagent

659 (Invitrogen 11668-030) and 2mg of each respective plasmid. Non-Homology End Joining 
660 (NHEJ) repair created a deletion around the gRNA cutting site. Cells were selected with

661 Puromycin for 2 days. Genomic DNA was extracted and the region around the cutting

662 site was PCR amplified. Knockout of the gene TAOK2 was confirmed by Sanger

663 sequencing analysis, and absence of encoded protein was validated using western blot.

\section{Cell Culture and Maintenance}

665 All experiments were performed in HEK293T cells, which were grown in DMEM media

666 (Thermo Fisher, Gibco) with 10\% fetal bovine serum (Axenia BioLogix) and 1\% Pen-

667 Strep (Invitrogen). Cells were maintained at $5 \% \mathrm{CO} 2$ and $37^{\circ} \mathrm{C}$ and passaged every $3-4$

668 days.

669 Immunofluorescence and Western Blotting

670 Cells were fixed with $4 \%$ paraformaldehyde and $4 \%$ sucrose for 20 minutes at room

671 temperature, followed by 3 washes with phosphate-buffered saline (PBS). One-hour

672 incubation with blocking buffer (200mM Glycine pH 7.4, 0.25\% TritonX-100, 10\% Normal

673 Donkey Serum, in PBS) was followed by overnight incubation with primary antibody at a

674 1:1000 dilution in blocking buffer. After three 5min washes in PBS, cells were incubated

675 with secondary antibody at 1:1000 dilution in blocking buffer for $3 \mathrm{hr}$. Coverslips were

676 washed and then mounted onto slides with FluoromontG. Endogenous TAOK2 staining

677 was performed similarly, except cells were fixed with cold methanol incubated on ice for

67820 minutes instead of PFA. Samples for western blot analysis were treated with 4X LDS

679 Sample Buffer (Thermo Fisher) with 125 mM DTT and subsequently heated for 10

680 minutes at 95C. Samples were electrophoresed on NuPAGE 4-12\% Bis-Tris

681 Polyacrylamide gels (Thermo Fisher) with NuPAGE MOPS running buffer (Thermo

682 Fisher). Western blot transfer to ImmobilonP PVDF membrane (Millipore-Sigma) with

683 Transfer Buffer (25mM Tris, 192mM Glycine, 20\% (v/v) Methanol, 0.05\% SDS) at 100V

684 for $60 \mathrm{~min}$. Resultant blot was blocked in 5\% milk or BSA blocking buffer, and subjected

685 to primary antibody and HRP conjugated secondary antibody before visualization with 
686 Pierce $^{\mathrm{TM}}$ ECL Western Blotting Substrate (Thermo Fisher). Western blot images were

687 obtained using the ChemiDoc Imager (BioRad).

688 Image Analysis and quantification

689 MT growth analysis was performed using the ImageJ plugin for manually tracking

690 objects (Manual Tracking). Single frame time lapse image stacks were processed to

691 measure the distance traveled by EB3 comets in each frame. Velocity was calculated by

692 dividing the total distance traveled by the time taken. Curvature was calculated by

693 dividing the minimum linear path (from beginning to end) by the length of the actual

694 distance the EB3 comet traveled. Time paused was calculated by measuring the number

695 of times the EB3 comet did not change coordinates multiplied by time between each

696 frame (2s). Percent time paused is calculated by divided by the total time the comet was

697 tracked.

$698 \quad$ ER motility was measured from single-z frame image stacks acquired from

699 imaging the ER markers (EGFP-Sec22 or ER-mRFP) using ImageJ. Substacks were

700 created corresponding to frames 6 s apart and pixel differences every 6 s were calculated

701 using the Stack Difference function to determine a change in fluorescence $(\Delta \mathrm{F}) . \Delta \mathrm{F}$ was

702 then divided by the mean fluorescence $(F)$ of the earliest time point frame from which it

703 was derived. (i.e., $\Delta F$ between frames 3 and 4 would be divided by frame 3) These $\Delta F / F$

704 values were taken for each time point and averaged over 2 minutes to determine ER

705 motility for each cell. To obtain the cumulative pixel difference the substack obtained

706 from using the Stack Difference function was z-projected with the 'sum slices' option,

707 and then pseudocolored using the physics LUT. This method to calculate ER motility

708 index is an adaptation from Dong et al. (2018) (Dong et al., 2018) with specified

709 changes.

710 To assess mitotic defects, four color images were acquired as z-stacks with

7110.3 micron spacing such that the entire mitotic cell was captured. Mitotic defects were 
712 scored manually by visualizing the entire $z$ stack, based on the spindle morphology, ER

713 morphology and chromosomal localization.

\section{Differential Centrifugation Assay}

715 HEK293T cells were grown to confluence in four $10 \mathrm{~cm}$ dishes using DMEM media with

$71610 \%$ fetal bovine serum and $1 \%$ Pen-Strep. Cells were washed once with Dulbecco's

717 PBS, collected in ice cold PBS and pelleted by centrifugation at $200 \mathrm{~g}$. Pellet was

718 resuspended in $2 \mathrm{~mL}$ of homogenization buffer (250mM sucrose, $10 \mathrm{mM}$ HEPES, $1 \mathrm{mM}$

719 EDTA, protease inhibitors (Roche), 1mM PMSF, and 1mM DTT) and homogenized with

720 a 25-gauge syringe needle. Homogenate was subsequently spun at $800 \mathrm{~g}$ to pellet

721 nuclear fraction. Post nuclear supernatant (S1) was diluted in homogenization buffer to

722 split between two $2 \mathrm{~mL}$ ultracentrifuge tubes. Heavy membrane fraction (P2) was

723 obtained by centrifuging $\mathrm{S} 1$ at $27,000 \mathrm{~g}$ for 30 minutes at $4{ }^{\circ} \mathrm{C}$. Light membrane fraction

724 (P3) was obtained by centrifuging S2 at 100,00g for 30 minutes. P3 was resuspended in

725 either 200uL: homogenization buffer (control), or detergent buffer (1\% NP-40, 1\%

726 TritonX-100, 0.1\% SDS) and incubated on ice for 30 minutes before spinning at

$727200,000 \mathrm{~g}$ for 60 minutes at $4^{\circ} \mathrm{C}$. Resultant high speed pellets (P4) were resuspended in

728 4x sample buffer with $125 \mathrm{mM}$ DTT. Resultant supernatants S4 and cytosolic supernatant

729 fraction S3 were precipitated with ice cold 10\% trichloroacetic acid by incubating on ice

730 for 15 minutes followed by centrifugation at $21,000 \mathrm{~g}$. Precipitates were washed with ice-

731 cold acetone, and pelleted at 21,000g for 5 minutes and resuspended in $4 \mathrm{x}$ sample

732 buffer with $125 \mathrm{mM}$ DTT. All samples were run on SDS-PAGE gels and transferred to

733 PVDF membrane for western blot analysis.

\section{ER Membrane Isolation}

735 HEK293T cells were grown to confluence in four $10 \mathrm{~cm}$ dishes. Cells were washed once

736 with Dulbecco's PBS, collected in ice cold PBS and pelleted by centrifugation at $200 \mathrm{~g}$.

737 Pellet was resuspended in $1 \mathrm{~mL}$ of isolation buffer $(225 \mathrm{mM}$ mannitol, $75 \mathrm{mM}$ sucrose, 
$73830 \mathrm{mM}$ Tris-HCl pH 7.4, 0.1mM EGTA and protease inhibitors (Roche), and

739 homogenized with a $25 \mathrm{G}$ needle at $4^{\circ} \mathrm{C}$. The homogenate was subsequently subjected

740 to a series of spins at $4^{\circ} \mathrm{C}$, retaining the pellet from each and continuing with supernatant

741 to the next spin. The centrifugation schema was as follows (adapted from Hoyer et al.

742 2018): 2 spins at $600 \mathrm{~g}$ for 5 minutes to pellet both cell debris and nuclei (P1 and P2), 3

743 spins at $7000 \mathrm{~g}$ for 5 minutes each to pellet mitochondria (P3, P4, and P5), and a

$74420,000 \mathrm{~g}$ spin for 20 minutes to pellet the crude ER fraction (P6). The supernatant from

745 the last spin yielded the cytosol, and P6 was washed with isolation buffer devoid of

746 EGTA and subject to a $20,000 \mathrm{~g}$ spin for 15 minutes at $4^{\circ} \mathrm{C}$ to re-pellet. P1-6 were

747 resuspended in resuspension buffer (50mM HEPES, $2.5 \mathrm{mM} \mathrm{MgCl}_{2}, 200 \mathrm{mM} \mathrm{KCl,} 5 \%$

748 glycerol, 1\% TritonX-100). Protein concentrations of resuspended P1-6 and cytosolic

749 fraction were quantified by BCA assay, and subsequently $4 \mathrm{X}$ sample buffer with $0.125 \mathrm{M}$

750 DTT was added. This method of crude organellar separation was adapted from Hoyer et

751 al., (2018) (Hoyer et al., 2018). Normalized samples were analyzed by western blot.

\section{Microscopy}

753 Superresolution imaging was performed using the Nikon-CSU-W1 Spinning Disk

754 equipped with a microlensed SoRa emission disk that achieves Super Resolution by

755 Optical Pixel Reassignment with a xy resolution of $120 \mathrm{~nm}$. Images were acquired on an

756 inverted Nikon Eclipse Ti2 microscope (Nikon Instruments) attached to a Yokogawa

757 spinning disk unit (CSU-W1 SoRa, Yokogawa Electric) using a 1.49 100x Apo TIRF oil

758 immersion objective lens. Images were captured by an Andor Sona 4.2B-11 camera

759 using the 2.8x SoRa relay, resulting in an effective pixel size of $\sim 40 \mathrm{~nm} .405,488$, and

$760561 \mathrm{~nm}$ laser lines were used for excitation. All other live and fixed cell imaging was

761 performed on a Nikon Ti2 Eclipse-CSU-X1 confocal spinning disk microscope equipped

762 with four laser lines $405 \mathrm{~nm}, 488 \mathrm{~nm}, 561 \mathrm{~nm}$ and $670 \mathrm{~nm}$ and an sCMOS Andor camera

763 for image acquisition. The microscope was caged within the OkoLab environmental 
764 control setup enabling temperature and $\mathrm{CO}_{2}$ control during live imaging. Imaging was

765 performed using Nikon 1.49 100x Apo 100X or 60X oil objectives. Live imaging for ER

766 motility and EB3 comet velocity was performed on fibronectin coated MatTek dishes

767 (MatTek, P35G-1.5-14-C), and images at a single confocal z frame were captured every

768 2sec. Fixed cell image acquisition was performed as a z stack of images with z distance

769 of 0.3 micron. Viafluor microtubule live imaging dyes (Biotum, \#70064, \#70063) were

770 used to visualize microtubules during live imaging. Cells were incubated for 30 minutes

771 at 37 degrees $C$ with (1:2000) dye in culture media. Subsequently, dye treated media is

772 replaced with live imaging media with (1:10000) tubulin dye. DNA was stained with

773 NucBlue ${ }^{\mathrm{TM}}$ Live ReadyProbes ${ }^{\mathrm{TM}}$ Reagent (Hoechst 33342) (Invitrogen), two drops/mL

774 live imaging media incubated for 15 minutes at room temperature before imaging.

\section{Protein Purification}

776 TAOK2 C-terminal amino acids 1187-1235 were cloned into pGEX4T1 vector and

777 transformed into BL21 E. coli to bacterially express the GST-tagged TAOK2 1187-1235.

778 A $25 \mathrm{ml}$ starter culture grown from a single colony overnight was used to inoculate $1 \mathrm{~L}$

779 culture, which was allowed to grow to an O.D, of 0.6 at $37^{\circ} \mathrm{C}$. Protein expression was

780 induced by IPTG at a final concentration of $0.4 \mathrm{mM}$ for 18 hours at $18^{\circ} \mathrm{C}$. Bacteria were

781 collected by a $15 \mathrm{~min}$ spin at $5000 \mathrm{~g}$, washed with ice cold PBS, and the pellet was

782 resuspended in ice cold lysis buffer (50mM Tris pH 8.0, 5mM EDTA, 150mM NaCl, 20\%

783 glycerol, 5mM DTT, protease inhibitors and PMSF). Addition of 4mg lysozyme (Sigma)

784 was followed by $30 \mathrm{~min}$ incubation with $0.5 \%$ TritonX-100 and sonication on ice. The

785 supernatant was collected after a $60 \mathrm{~min}$ spin at $25,000 \mathrm{~g}$, and incubated with prewashed

786 GST beads (Thermo Fisher) for 1 hour. Beads were washed with wash buffer (PBS +

$7871 \mathrm{mM}$ DTT $+0.1 \%$ tween 20 ) followed by wash buffer without detergent. Bound protein 
788 was eluted and collected in fractions by glutathione elution buffer at pH8.0 (50mM Tris

$789 \mathrm{pH} 8.0,250 \mathrm{mM} \mathrm{KCl}, 1 \mathrm{mM}$ DTT, 10\% glycerol and 30mM glutathione).

\section{Microtubule co-sedimentation assay}

791 Microtubules were prepared by polymerizing porcine tubulin (Cytoskeleton inc.) in

792 general tubulin buffer (80mM PIPES pH 6.9, 2mM MgCl, $0.4 \mathrm{mM}$ EGTA, Roche

793 Protease Inhibitors) in the presence of $1 \mathrm{mM}$ GTP for 20 minutes at $35^{\circ} \mathrm{C}$ and then

794 diluted further. To prevent depolymerization, microtubules were treated with $40 \mu \mathrm{M}$

795 Taxol. Microtubules and $5 \mu \mathrm{g}$ purified protein were incubated at room temperature, and

796 pelleted at 100,000g over a 60\% glycerol cushion buffer (80mM PIPES pH 7.0, 1 mM

$797 \mathrm{MgCl}$, $1 \mathrm{mM}$ EGTA, 60\% Glycerol, protease inhibitor). The supernatant (top layer

798 above cushion) and the pellet were removed and treated with 4X Sample Buffer with

$799250 \mathrm{mM}$ DTT and 5\% beta-mercaptoethanol. Resultant samples were subject to SDS-

800 PAGE and colloidal Coomassie blue staining (Invitrogen).

$801 \quad$ Mitotic cell lysate

802 HEK293T cells were synchronized by treatment with $1.67 \mu \mathrm{M}$ nocodazole for $12-16$

803 hours. Rounded cells were dislodged by shaking and collected with media. Concurrently,

804 untreated asynchronously growing HEK293T cells were scraped and collected in DPBS.

805 Both tubes of cells were separately pelleted, washed in DPBS, and lysed in HKT buffer

806 (25mM HEPES pH7.2, 150mM KCl, 1\% Triton X-100, 1mM DTT, 1 mM EDTA, Protease

807 Inhibitors (Roche, Complete), Halt Phosphatase Inhibitors (Thermo Fisher). Lysate was

808 cleared of cell debris by centrifugation and protein concentrations were determined via

809 BCA assay (Thermo Fisher). Sample Buffer with 125mM DTT was added to equalized

810 amounts of protein and subject to western blot analysis as described above.

\section{Immunoprecipitation Kinase Assay}

812 HEK293T cells transfected with sfGFP-TAOK2 WT and sfGFP-TAOK2 K57A were lysed 813 with HKT buffer (25mM HEPES pH7.2, 150mM KCl, 1\% Triton X-100, 1mM DTT, $1 \mathrm{mM}$ 
814 EDTA, Protease Inhibitors (Roche, Complete)). Lysate was precleared with Pierce

815 ProteinG Agarose (Thermo Fisher), and immunoprecipitated with Roche anti-GFP

816 Mouse antibody bound on Pierce protein G agarose. Beads were washed twice with

817 HKT, once for 10 minutes with HKT with $1 \mathrm{mM} \mathrm{NaCl}$, and finally washed with HK buffer

818 (25mM HEPES pH 7.2, 150mM KCl, 1mM DTT, 1 mM EDTA, Protease Inhibitors

819 (Roche, Complete EDTA free)). Beads were washed once with the Kinase Buffer (25mM

820 Tris $\mathrm{pH} 7.5,10 \mathrm{mM} \mathrm{MgCl} 2,1 \mathrm{mM}$ DTT) prior to the in vitro kinase assay. Kinase assay

821 was then performed by incubating with $0.5 \mathrm{mM}$ ATP and Halt protease/phosphatase

822 inhibitors (Thermo Fisher) for 45 minutes at $30^{\circ} \mathrm{C}$ on a shaking heat block. Samples

823 were then subjected to western blot analysis detailed above to detect

824 autophosphorylation of TAOK2 at S181 using the rabbit antibody (R\&D Systems,

825 PPS037).

\section{Statistics}

827 All statistics were performed in GraphPad software Prism9.0. Multiple groups were

828 analyzed using ANOVA, while two group comparisons were made using unpaired t-test

829 unless otherwise stated. Statistically $p$ value less that 0.05 was considered significant.

830 All experiments were done in triplicate, and experimental sample size and $p$ values are

831 indicated with the corresponding figures. 


\section{References}

835 Carlton, J.G., Jones, H., and Eggert, U.S. (2020). Membrane and organelle dynamics 836 during cell division. Nat. Rev. Mol. Cell Biol. 21, 151-166.

837 Chen, Z., Hutchison, M., and Cobb, M.H. (1999). Isolation of the protein kinase TAO2 838 and identification of its mitogen-activated protein kinase/extracellular signal-regulated 839 kinase kinase binding domain. J. Biol. Chem. 274, 28803-28807.

840 Chen, Z., Raman, M., Chen, L., Lee, S.F., Gilman, A.G., and Cobb, M.H. (2003). TAO 841 (thousand-and-one amino acid) protein kinases mediate signaling from carbachol to p38 842 mitogen-activated protein kinase and ternary complex factors. J. Biol. Chem. 278, $84322278-22283$.

844 Combet, C., Blanchet, C., Geourjon, C., and Deléage, G. (2000). NPS@: network 845 protein sequence analysis. Trends Biochem Sci 25, 147-150.

846 Cox, J.S., Shamu, C.E., and Walter, P. (1993). Transcriptional induction of genes 847 encoding endoplasmic reticulum resident proteins requires a transmembrane protein 848 kinase. Cell 73, 1197-1206.

849 Cui-Wang, T., Hanus, C., Cui, T., Helton, T., Bourne, J., Watson, D., Harris, K.M., and 850 Ehlers, M.D. (2012). Local zones of endoplasmic reticulum complexity confine cargo in 851 neuronal dendrites. Cell 148, 309-321.

852 de Anda, F.C., Rosario, A.L., Durak, O., Tran, T., Gräff, J., Meletis, K., Rei, D., Soda, T., 853 Madabhushi, R., Ginty, D.D., et al. (2012). Autism spectrum disorder susceptibility gene 854 TAOK2 affects basal dendrite formation in the neocortex. Nat. Neurosci. 15, 1022-1031.

855 Dong, R., Zhu, T., Benedetti, L., Gowrishankar, S., Deng, H., Cai, Y., Wang, X., Shen, 856 K., and De Camilli, P. (2018). The inositol 5-phosphatase INPP5K participates in the fine 857 control of ER organization. J. Cell Biol. 217, 3577-3592.

858 Farías, G.G., Fréal, A., Tortosa, E., Stucchi, R., Pan, X., Portegies, S., Will, L., Altelaar, 859 M., and Hoogenraad, C.C. (2019). Feedback-Driven Mechanisms between Microtubules 860 and the Endoplasmic Reticulum Instruct Neuronal Polarity. Neuron 102, 184-201.e188.

861 Friedman, J.R., Webster, B.M., Mastronarde, D.N., Verhey, K.J., and Voeltz, G.K. 862 (2010). ER sliding dynamics and ER-mitochondrial contacts occur on acetylated 863 microtubules. J. Cell Biol. 190, 363-375.

864 Gautier, R., Douguet, D., Antonny, B., and Drin, G. (2008). HELIQUEST: a web server to 865 screen sequences with specific alpha-helical properties. Bioinformatics 24, 2101-2102.

866 Grigoriev, I., Gouveia, S.M., van der Vaart, B., Demmers, J., Smyth, J.T., Honnappa, S., 867 Splinter, D., Steinmetz, M.O., Putney, J.W., Hoogenraad, C.C., et al. (2008). STIM1 is a 868 MT-plus-end-tracking protein involved in remodeling of the ER. Curr. Biol. 18, 177-182. 
869 Harding, H.P., Zhang, Y., and Ron, D. (1999). Protein translation and folding are coupled by an endoplasmic-reticulum-resident kinase. Nature 397, 271-274.

871 Hazan, J., Fonknechten, N., Mavel, D., Paternotte, C., Samson, D., Artiguenave, F., 872 Davoine, C.S., Cruaud, C., Dürr, A., Wincker, P., et al. (1999). Spastin, a new AAA 873 protein, is altered in the most frequent form of autosomal dominant spastic paraplegia. 874 Nat. Genet. 23, 296-303.

875 Hoyer, M.J., Chitwood, P.J., Ebmeier, C.C., Striepen, J.F., Qi, R.Z., Old, W.M., and 876 Voeltz, G.K. (2018). A Novel Class of ER Membrane Proteins Regulates ER-Associated 877 Endosome Fission. Cell 175, 254-265.e14.

878 Hu, J., Shibata, Y., Zhu, P.-P., Voss, C., Rismanchi, N., Prinz, W.A., Rapoport, T.A., and 879 Blackstone, C. (2009). A class of dynamin-like GTPases involved in the generation of 880 the tubular ER network. Cell 138, 549-561.

881 Jongsma, M.L.M., Berlin, I., and Neefjes, J. (2015). On the move: organelle dynamics 882 during mitosis. Trends Cell Biol. 25, 112-124.

883 Klopfenstein, D.R., Kappeler, F., and Hauri, H.P. (1998). A novel direct interaction of 884 endoplasmic reticulum with microtubules. Embo J. 17, 6168-6177.

885 Krogh, A., Larsson, B., Heijne, von, G., and Sonnhammer, E.L. (2001). Predicting transmembrane protein topology with a hidden Markov model: application to complete genomes. J Mol Biol 305, 567-580.

888 Manning, G., Plowman, G.D., Hunter, T., and Sudarsanam, S. (2002). Evolution of protein kinase signaling from yeast to man. Trends Biochem Sci 27, 514-520.

890 Mitsopoulos, C., Zihni, C., Garg, R., Ridley, A.J., and Morris, J.D.H. (2003). The 891 prostate-derived sterile 20-like kinase (PSK) regulates microtubule organization and 892 stability. J. Biol. Chem. 278, 18085-18091.

893 Moore, T.M., Garg, R., Johnson, C., Coptcoat, M.J., Ridley, A.J., and Morris, J.D. 894 (2000). PSK, a novel STE20-like kinase derived from prostatic carcinoma that activates 895 the c-Jun N-terminal kinase mitogen-activated protein kinase pathway and regulates 896 actin cytoskeletal organization. J. Biol. Chem. 275, 4311-4322.

897 Mori, K., Ma, W., Gething, M.J., and Sambrook, J. (1993). A transmembrane protein with 898 a cdc2+/CDC28-related kinase activity is required for signaling from the ER to the 899 nucleus. Cell 74, 743-756.

900 Nikonov, A.V., Hauri, H.-P., Lauring, B., and Kreibich, G. (2007). Climp-63-mediated 901 binding of microtubules to the ER affects the lateral mobility of translocon complexes. J. 902 Cell. Sci. 120, 2248-2258.

903 Orso, G., Pendin, D., Liu, S., Tosetto, J., Moss, T.J., Faust, J.E., Micaroni, M., Egorova, 904 A., Martinuzzi, A., McNew, J.A., et al. (2009). Homotypic fusion of ER membranes 905 requires the dynamin-like GTPase atlastin. Nature 460, 978-983. 
906 Pavez, M., Thompson, A.C., Arnott, H.J., Mitchell, C.B., D'Atri, I., Don, E.K., Chilton,

907 J.K., Scott, E.K., Lin, J.Y., Young, K.M., et al. (2019). STIM1 Is Required for Remodeling

908 of the Endoplasmic Reticulum and Microtubule Cytoskeleton in Steering Growth Cones.

909 J. Neurosci. 39, 5095-5114.

910 Pendin, D., McNew, J.A., and Daga, A. (2011). Balancing ER dynamics: shaping,

911 bending, severing, and mending membranes. Curr Opin Cell Biol 23, 435-442.

912 Raman, M., Earnest, S., Zhang, K., Zhao, Y., and Cobb, M.H. (2007). TAO kinases

913 mediate activation of p38 in response to DNA damage. Embo J. 26, 2005-2014.

914 Richter, M., Murtaza, N., Scharrenberg, R., White, S.H., Johanns, O., Walker, S., Yuen,

915 R.K.C., Schwanke, B., Bedürftig, B., Henis, M., et al. (2018). Altered TAOK2 activity

916 causes autism-related neurodevelopmental and cognitive abnormalities through RhoA

917 signaling. Mol. Psychiatry 20, 1237.

918 Roll-Mecak, A., and Vale, R.D. (2008). Structural basis of microtubule severing by the 919 hereditary spastic paraplegia protein spastin. Nature 451, 363-367.

920 Ron, D., and Walter, P. (2007). Signal integration in the endoplasmic reticulum unfolded 921 protein response. Nat. Rev. Mol. Cell Biol. 8, 519-529.

922 Schlaitz, A.-L., Thompson, J., Wong, C.C.L., Yates, J.R., and Heald, R. (2013).

923 REEP3/4 ensure endoplasmic reticulum clearance from metaphase chromatin and

924 proper nuclear envelope architecture. Dev. Cell 26, 315-323.

925 Scorrano, L., De Matteis, M.A., Emr, S., Giordano, F., Hajnóczky, G., Kornmann, B.,

926 Lackner, L.L., Levine, T.P., Pellegrini, L., Reinisch, K., et al. (2019). Coming together to

927 define membrane contact sites. Nat Commun 10,1-11.

928 Smyth, J.T., DeHaven, W.I., Bird, G.S., and Putney, J.W. (2007). Role of the microtubule 929 cytoskeleton in the function of the store-operated Ca2+ channel activator STIM1. J. Cell.

930 Sci. 120, 3762-3771.

931 Smyth, J.T., Schoborg, T.A., Bergman, Z.J., Riggs, B., and Rusan, N.M. (2015). Proper

932 symmetric and asymmetric endoplasmic reticulum partitioning requires astral

933 microtubules. Open Biol 5, 150067.

934 Terasaki, M., and Reese, T.S. (1994). Interactions among endoplasmic reticulum, 935 microtubules, and retrograde movements of the cell surface. Cell Motil. Cytoskeleton 29, 936 291-300.

937 Terasaki, M., Chen, L.B., and Fujiwara, K. (1986). Microtubules and the endoplasmic 938 reticulum are highly interdependent structures. J. Cell Biol. 103, 1557-1568.

939 Ultanir, S.K., Yadav, S., Hertz, N.T., Oses-Prieto, J.A., Claxton, S., Burlingame, A.L., 940 Shokat, K.M., Jan, L.Y., and Jan, Y.-N. (2014). MST3 Kinase Phosphorylates TAO1/2 to 941 Enable Myosin Va Function in Promoting Spine Synapse Development. Neuron 84, 968942982. 
943 Vedrenne, C., Klopfenstein, D.R., and Hauri, H.-P. (2005). Phosphorylation controls

944 CLIMP-63-mediated anchoring of the endoplasmic reticulum to microtubules. Mol. Biol.

945 Cell 16, 1928-1937.

946 Voeltz, G.K., Rolls, M.M., and Rapoport, T.A. (2002). Structural organization of the

947 endoplasmic reticulum. EMBO Rep. 3, 944-950.

948 Wang, S., Tukachinsky, H., Romano, F.B., and Rapoport, T.A. (2016). Cooperation of 949 the ER-shaping proteins atlastin, lunapark, and reticulons to generate a tubular 950 membrane network. Elife 5, 209.

951 Waterman-Storer, C.M., and Salmon, E.D. (1998). Endoplasmic reticulum membrane 952 tubules are distributed by microtubules in living cells using three distinct mechanisms. 953 Curr. Biol. 8, 798-806.

954 Wu, H., and Voeltz, G.K. (2021). Reticulon-3 Promotes Endosome Maturation at ER 955 Membrane Contact Sites. Dev. Cell 56, 52-66.e57.

956 Wu, H., Carvalho, P., and Voeltz, G.K. (2018). Here, there, and everywhere: The 957 importance of ER membrane contact sites. Science 361, eaan5835.

958 Yadav, S., Oses-Prieto, J.A., Peters, C.J., Zhou, J., Pleasure, S.J., Burlingame, A.L., 959 Jan, L.Y., and Jan, Y.-N. (2017). TAOK2 Kinase Mediates PSD95 Stability and Dendritic 960 Spine Maturation through Septin7 Phosphorylation. Neuron 93, 379-393. 
bioRxiv preprint doi: https://doi.org/10.1101/2021.04.22.440958; this version posted April 22, 2021. The copyright holder for this preprint (which

A. Merge

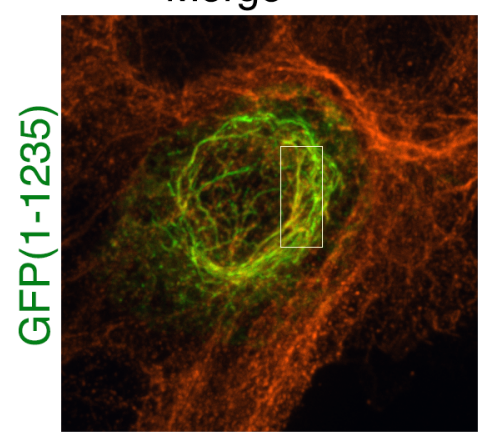

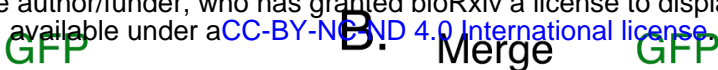
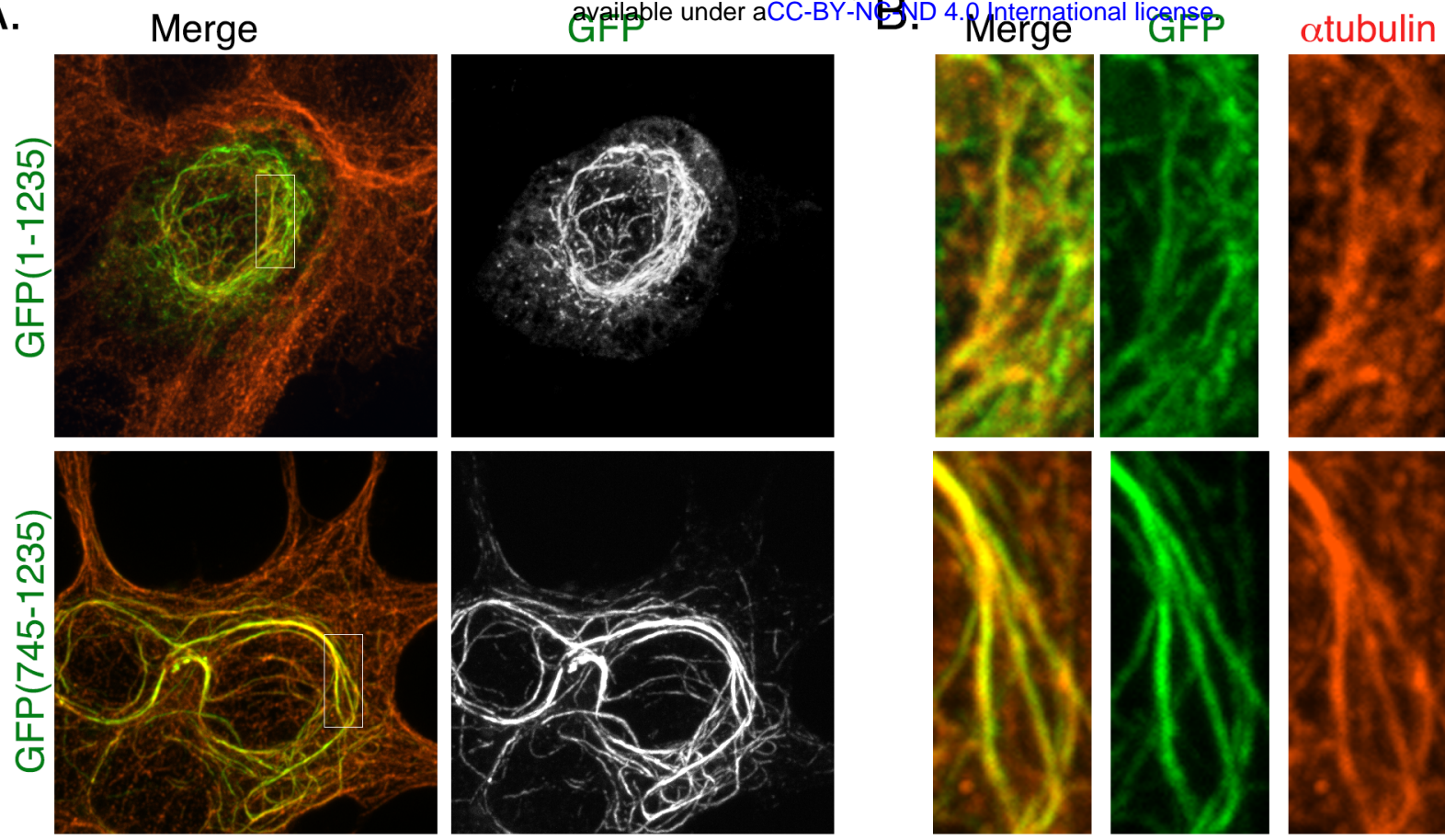

RGB Profile
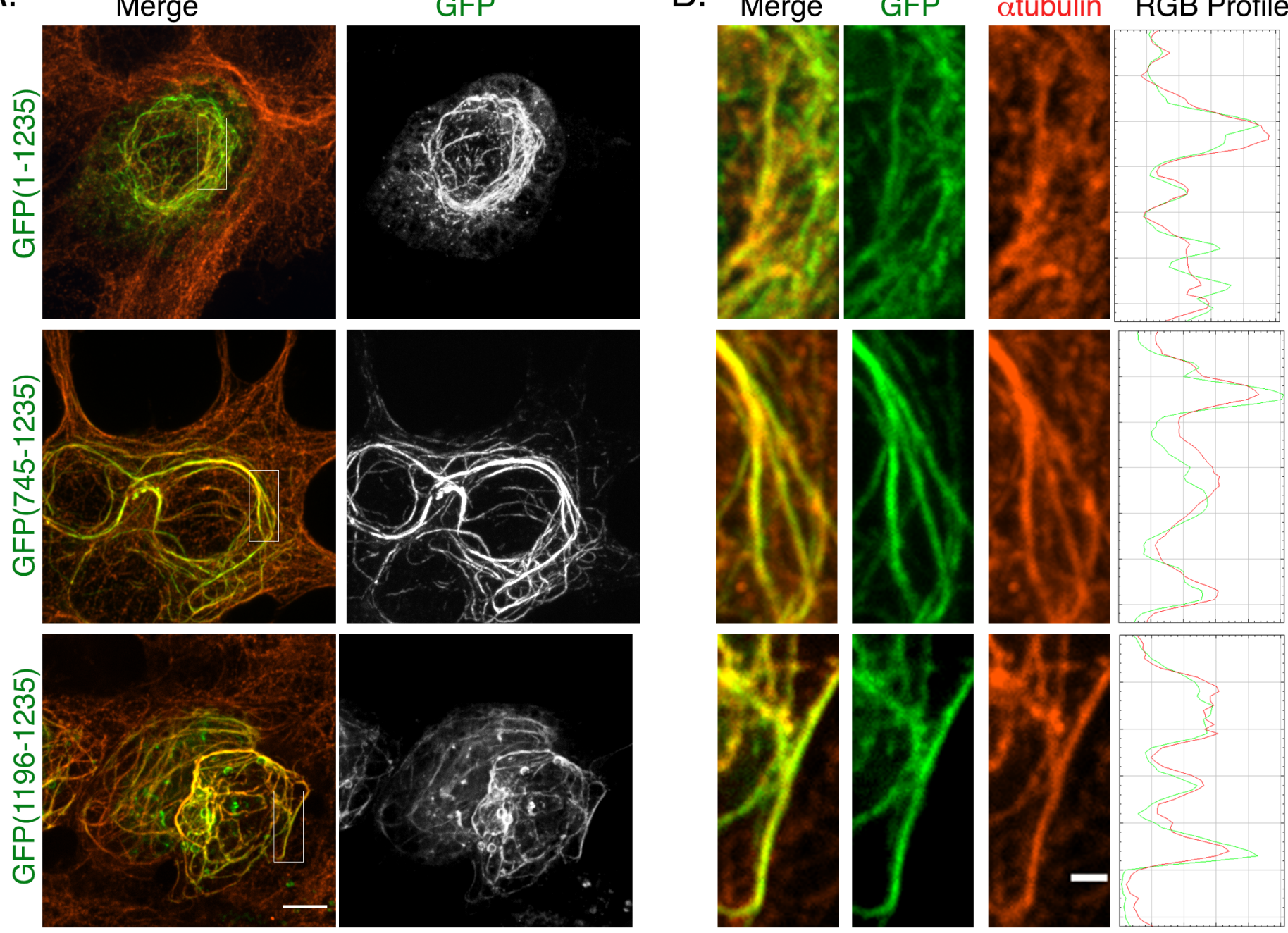

C.

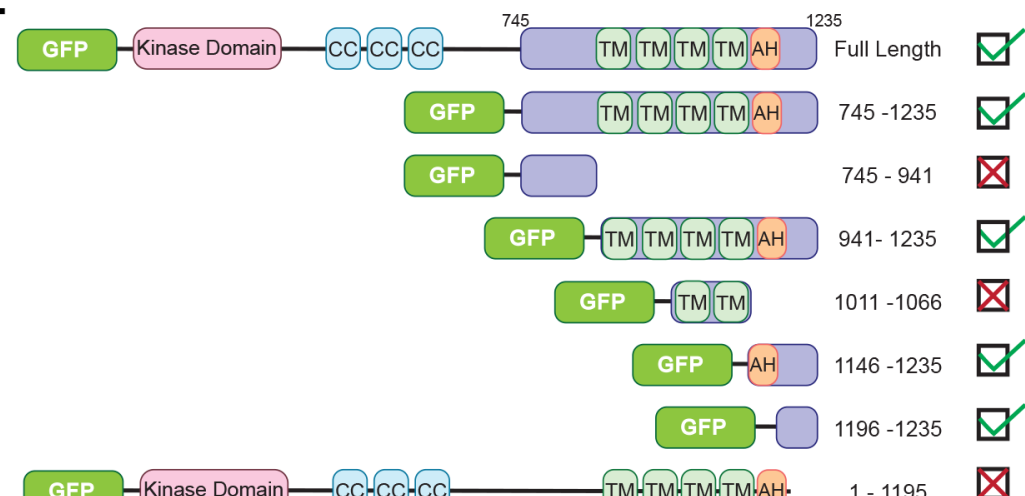

D.

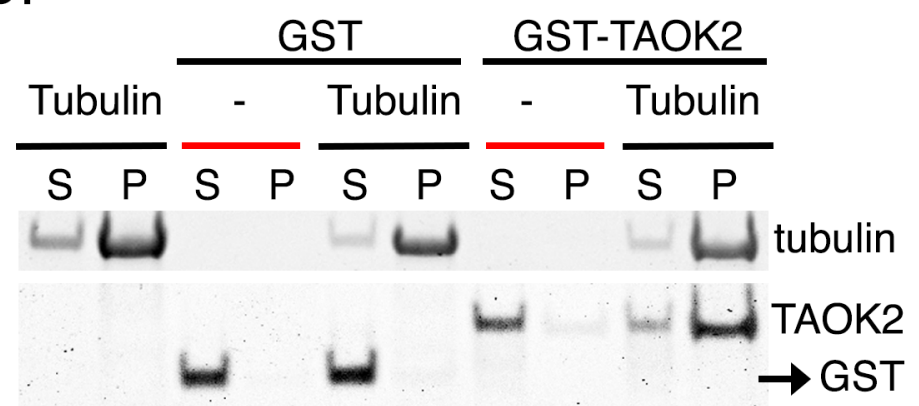

E

F.

G.

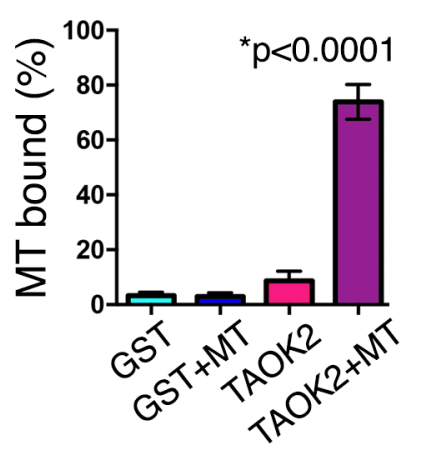

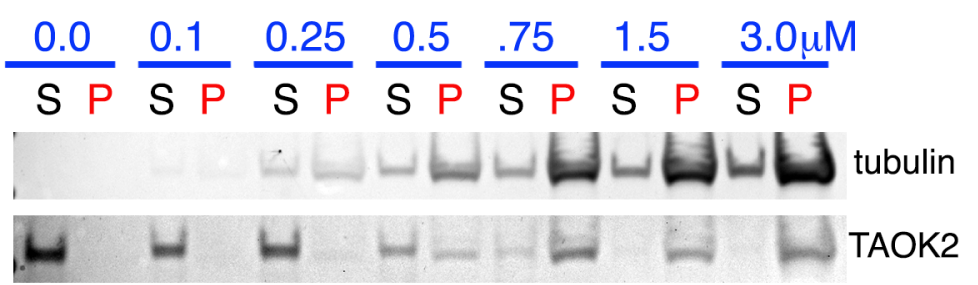

Coomaisse

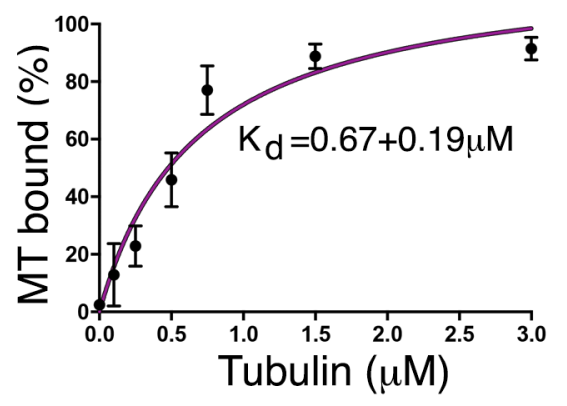


bioRxiv preprint doi: https://doi.org/10.1101/2021.04.22.440958; this version posted April 22, 2021. The copyright holder for this preprint (which

A.

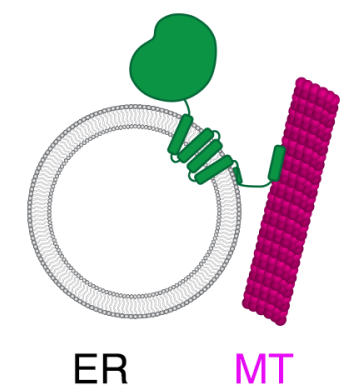

Merage
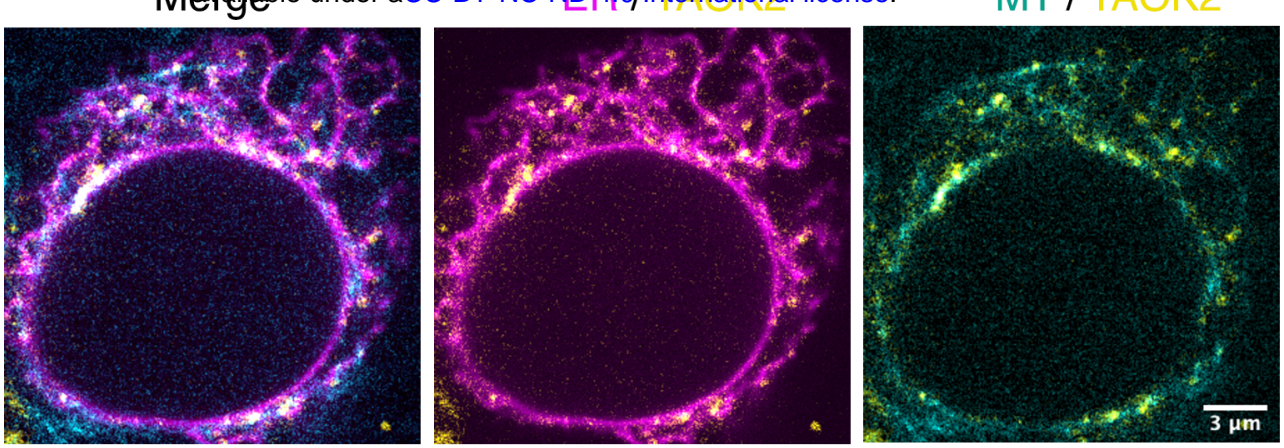

B.

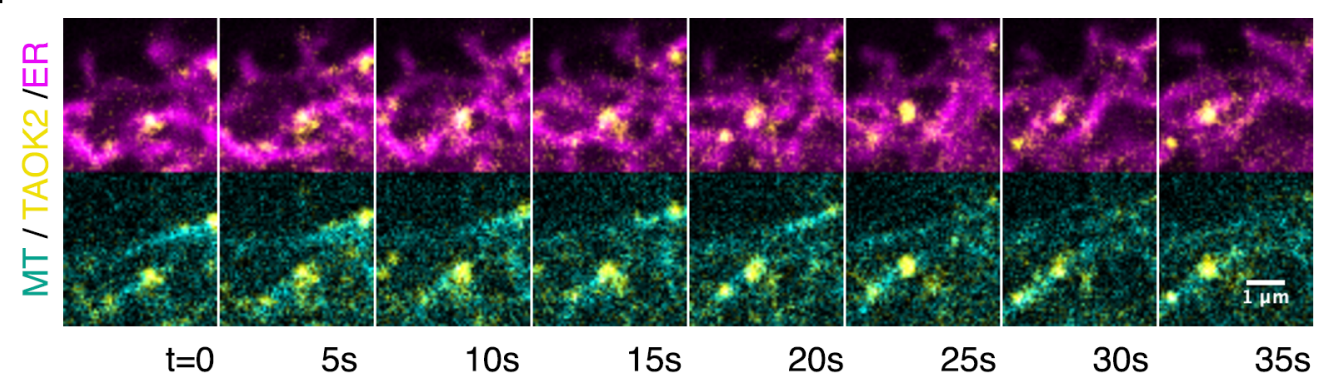

C.

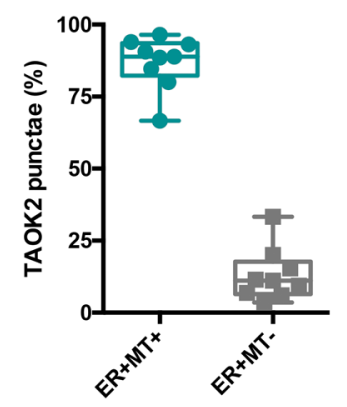

D.

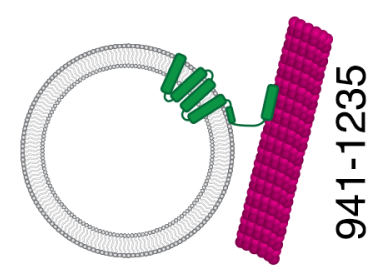

\section{ER}
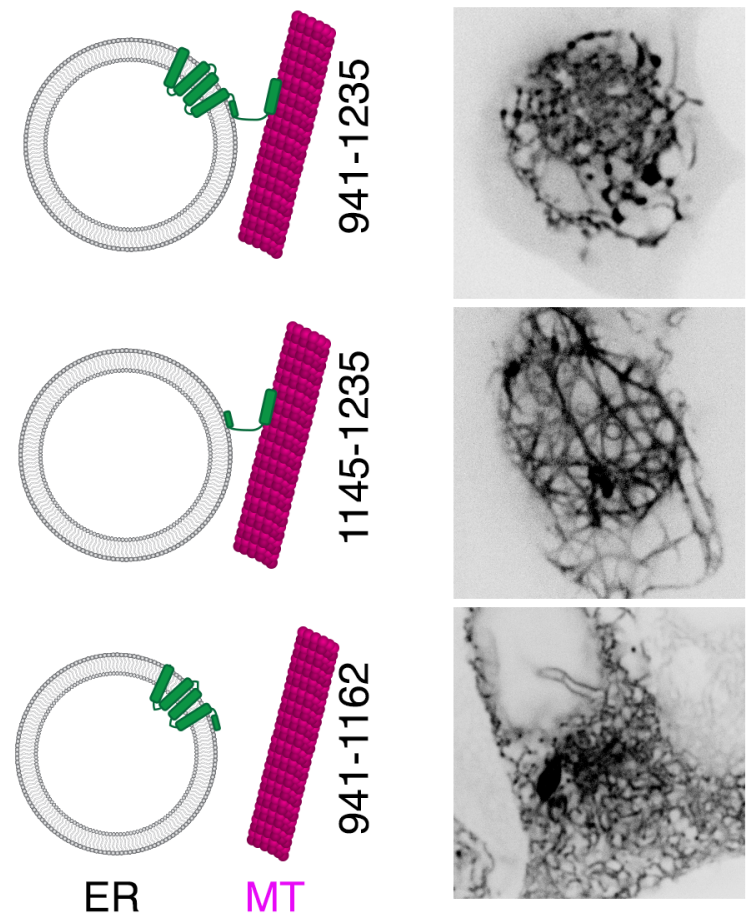

ER / TAOK2
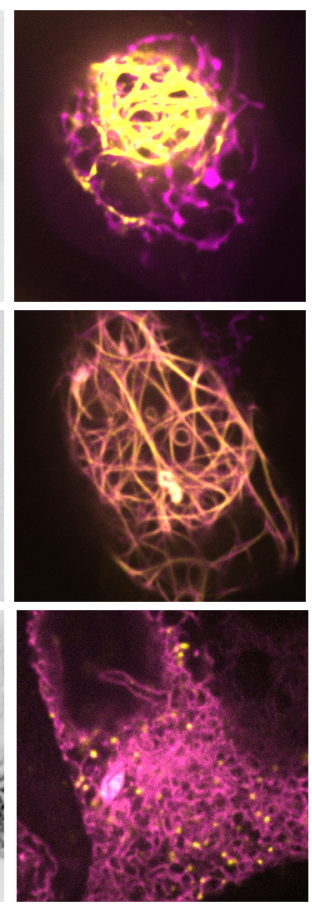
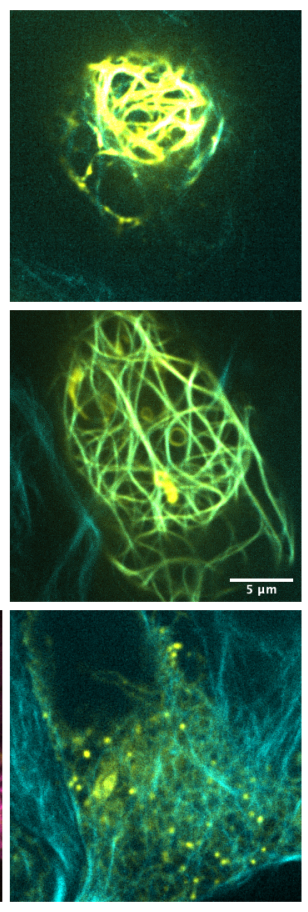

Figure 3 
A.

bioRxiv preprint doi: https://doi.org/10.1101/2021.04.22.440958; this version posted April 22, 2021. The copyright holder for this preprint (which WTailabl TAOK2 K KO

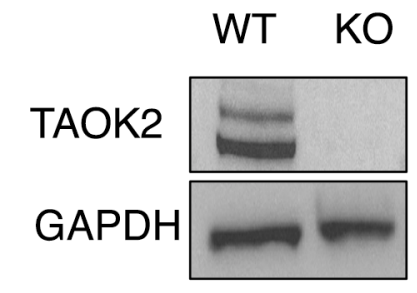

GAAATGGCAAGACATCATCAAGGAGGTGC TTCAGTACCGGGGCTGTTACCTGAGGGAG
Bientions
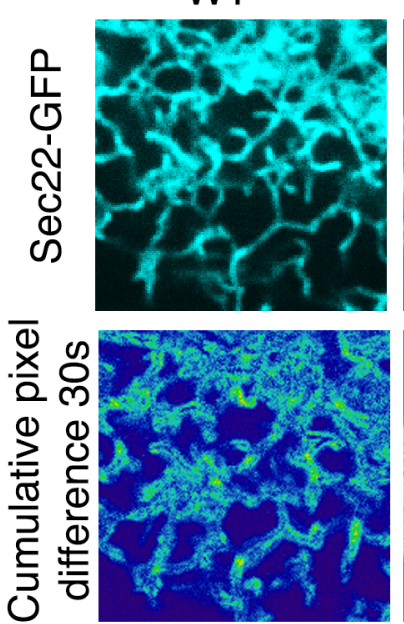
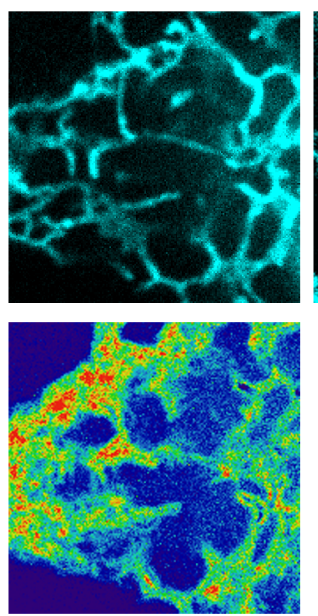
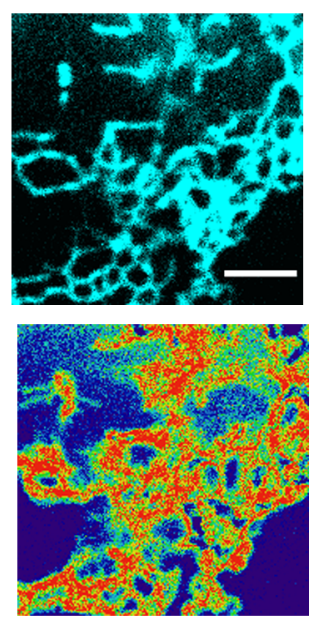
c.

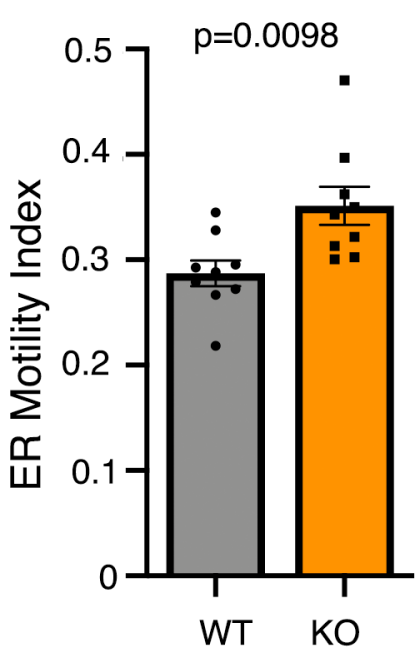

D.

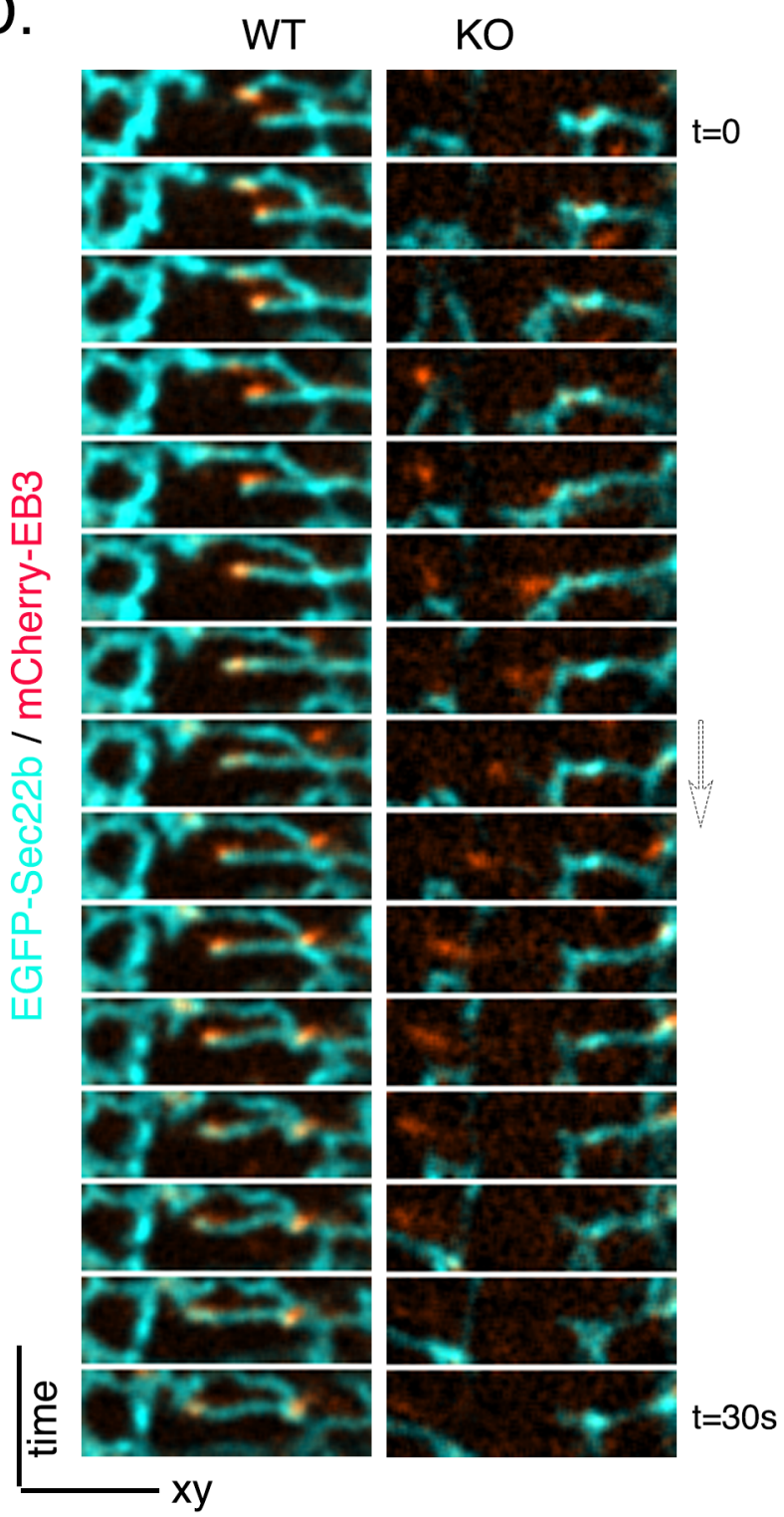

E.

Sliding event $\square$ ER TAC event

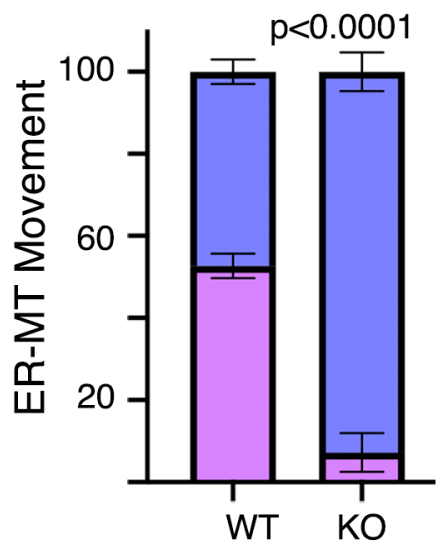

G.

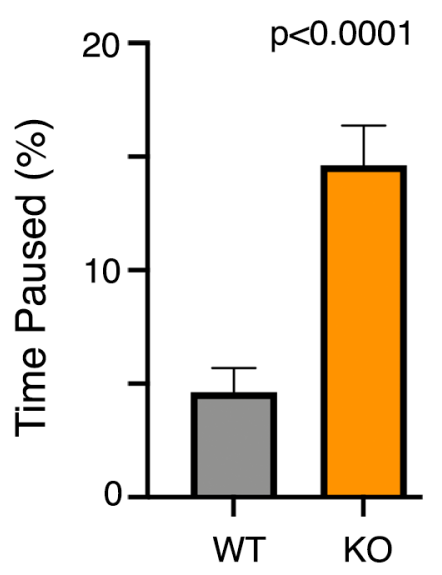

F. $p<0.0001$

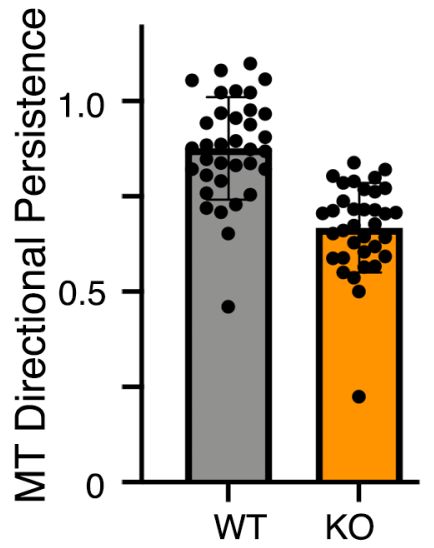

$\mathrm{H}$.

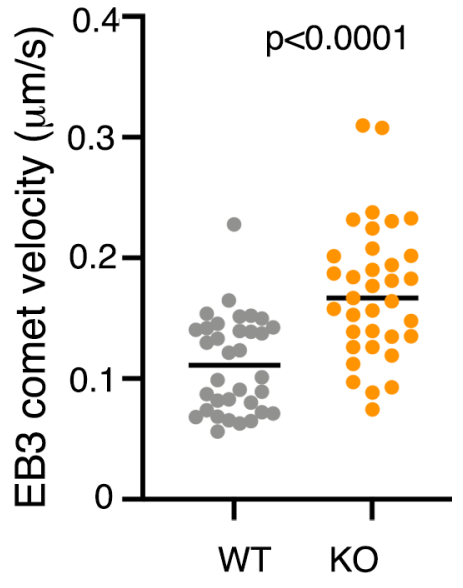

Figure 4 
bioRxiv preprint doi: https://doi.org/10.1101/2021.04.22.440958; this version posted April 22, 2021. The copyright holder for this preprint (which

A. was not certified by peer review) is the author/funder, who has granted bioRxiv a license to display the preprint in perpetuity. It is made
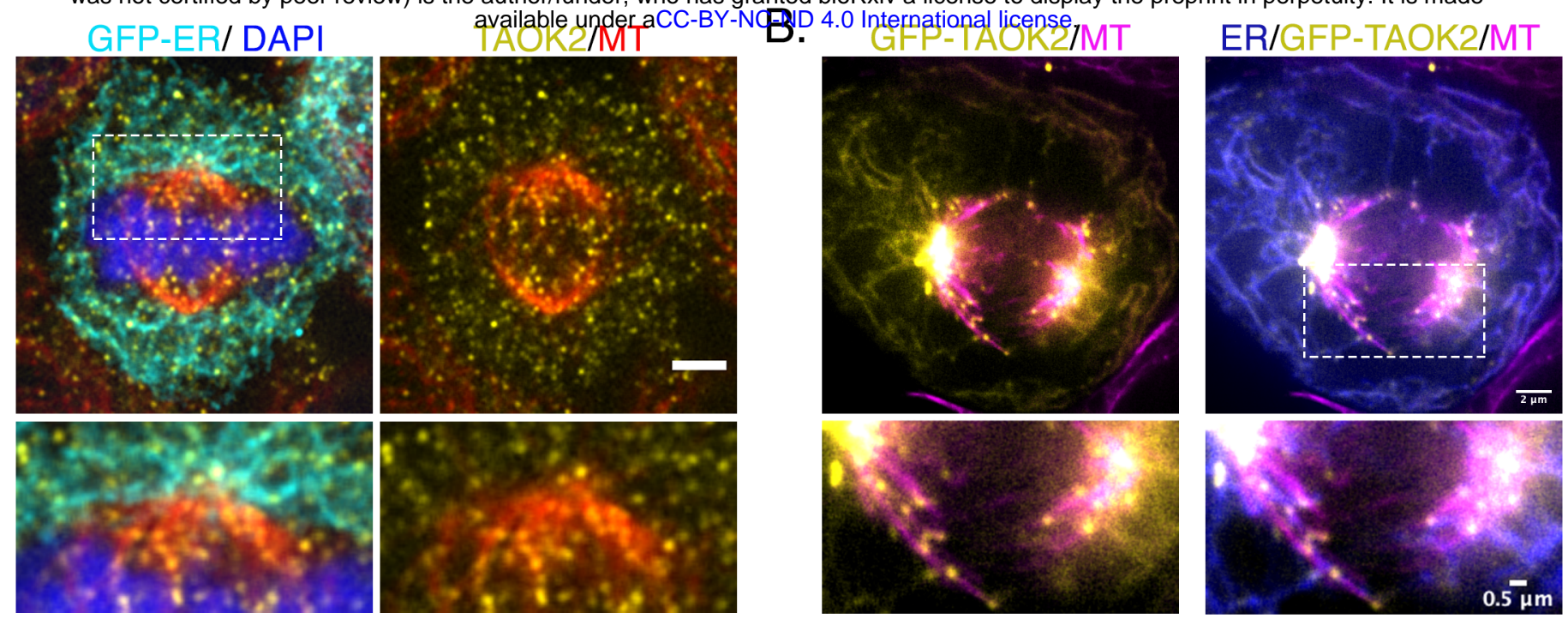

C

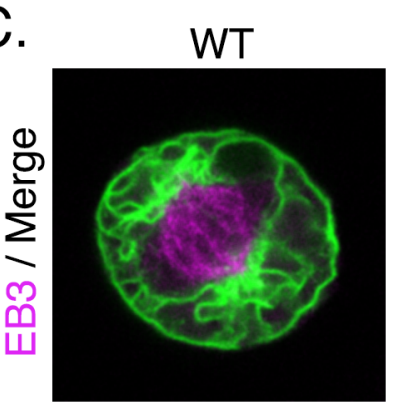

TAOK2-KO

D.

E.
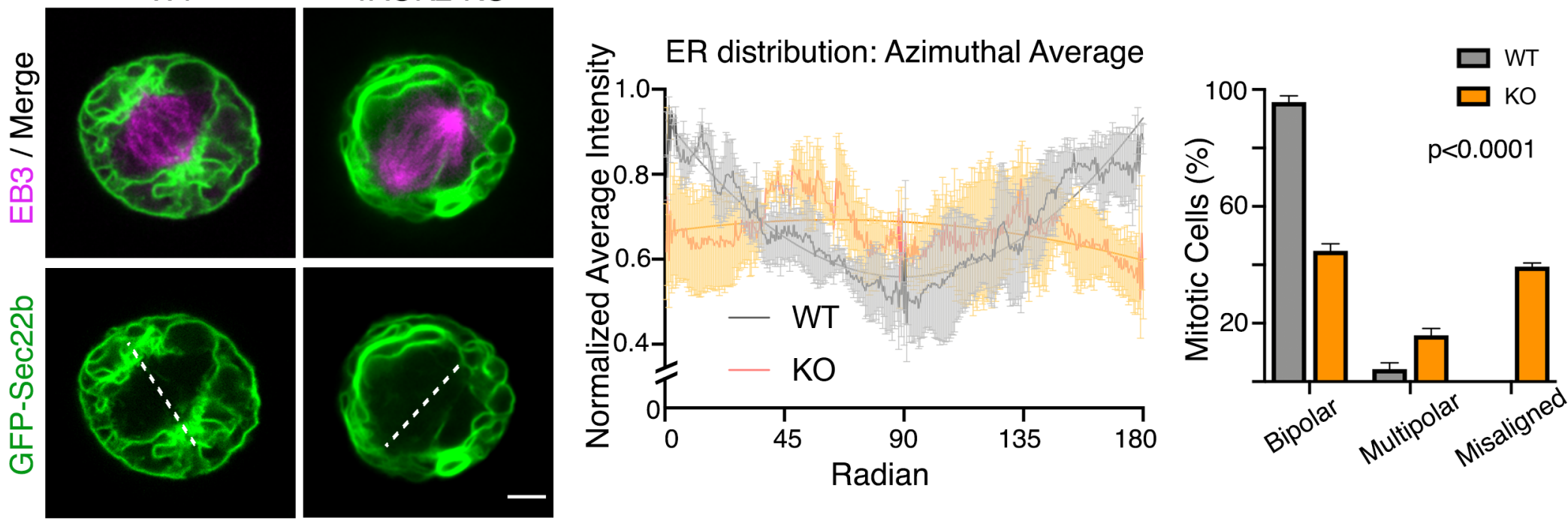
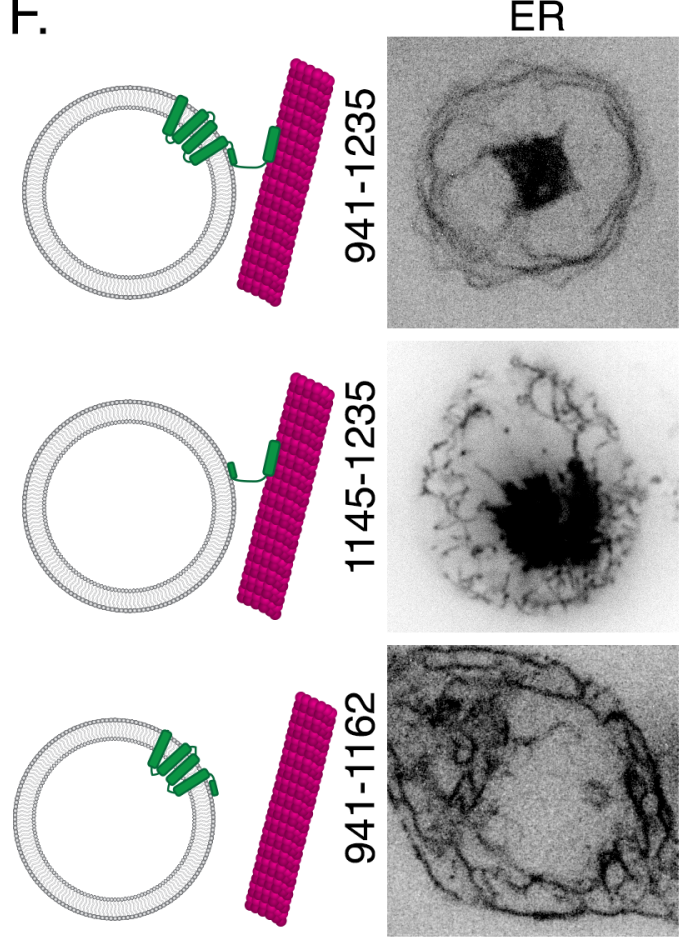
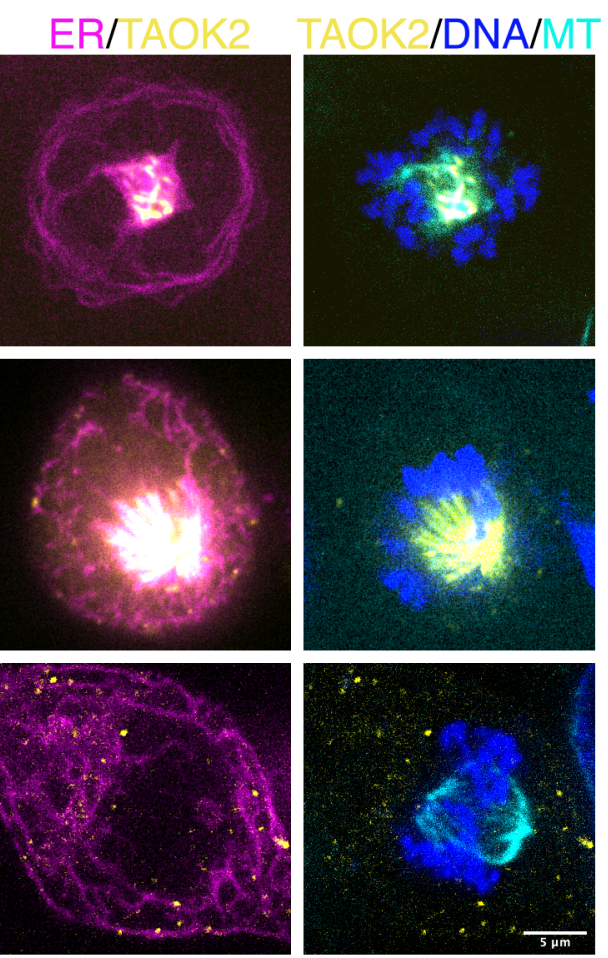

G.
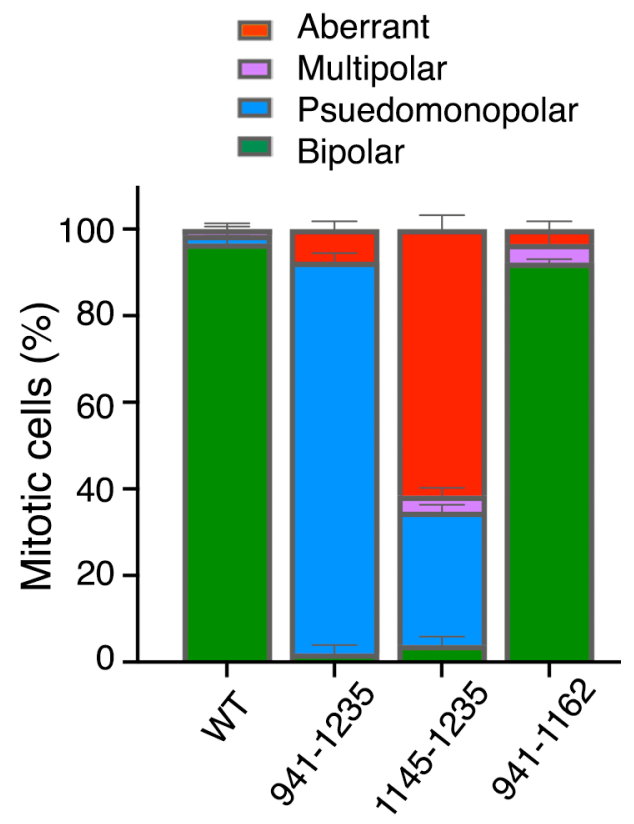
bioRxiv preprint doi: https://doi.org/10.1101/2021.04.22.440958; this version posted April 22, 2021. The copyright holder for this preprint (which was not certified by peer review) is the author/funder, who has granted bioRxiv a license to display the preprint in perpetuity. It is made available under aCC-BY-NC-ND 4.0 International license.

A.
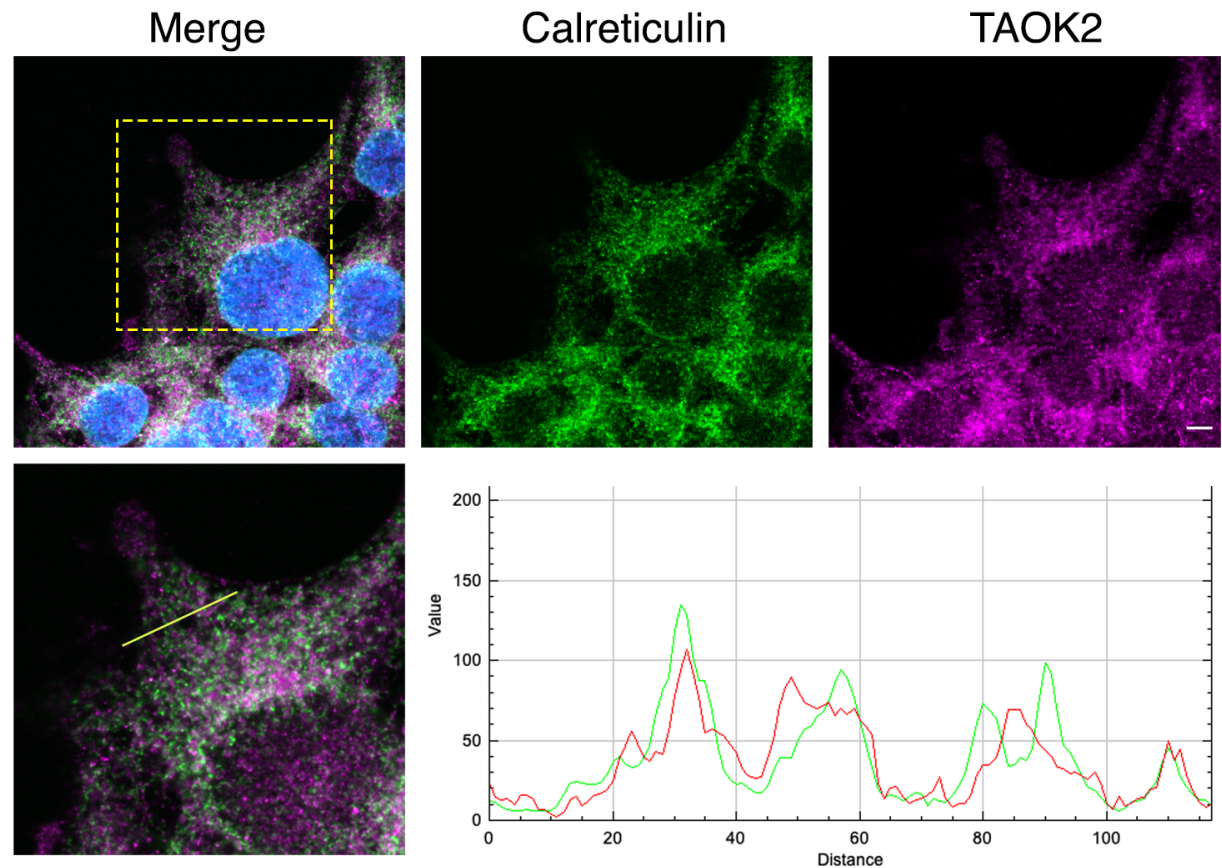

B.

\section{EGFP-Sec22 / TAOK2}
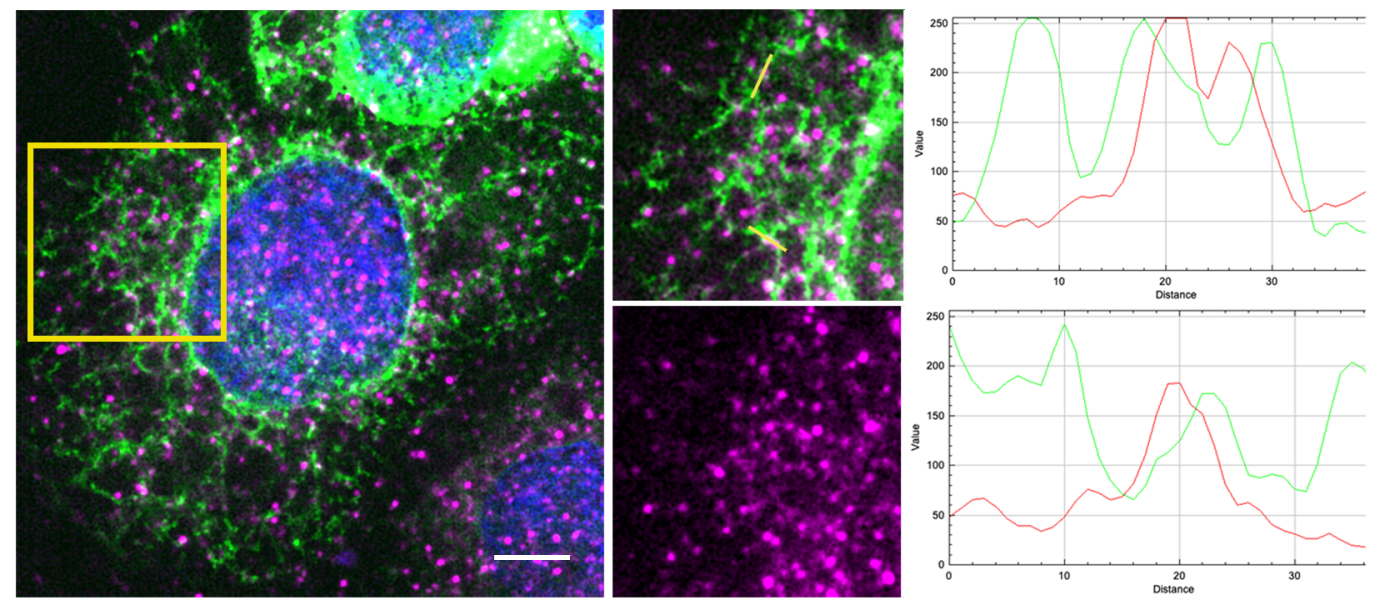

Figure S1 
bioRxiv preprint doi: https://doi.org/10.1101/2021.04.22.440958; this version posted April 22, 2021. The copyright holder for this preprint (which A. was not certified by peer review) is the author/funder, who has granted bioRxiv a license to display the preprint in perpetuity. It is made GST available under aCC-BYST-TÁNR 4.0 lnternational license.
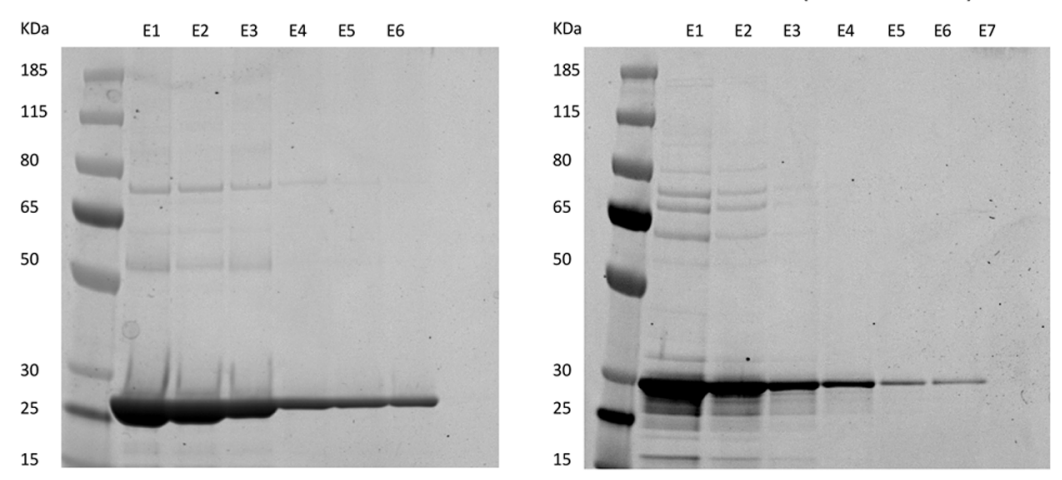

B.

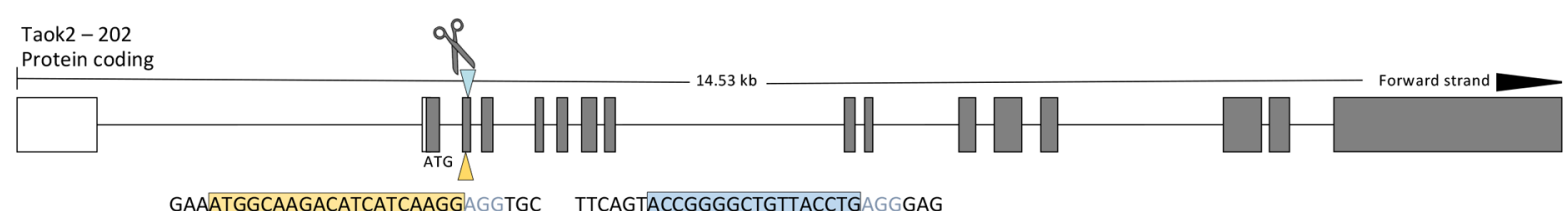

Mutation Pattern

WT САTCATCAAGGAGGTGCGGTTCTTACAGAAGCTCCGGCATCCCAACACCATTCAGTACCGGGGCTGTTACCTGAGGGAGCACACGGCTTGG КО САТСАТСА CC'T̄ÄÄGGAGCACACGGCTTGG

C.

WT

T GACCC C C TAG AAATGGCAAGACATCATCAAGGAGGTGCG GTTCTTACA

TAOK2 KO1

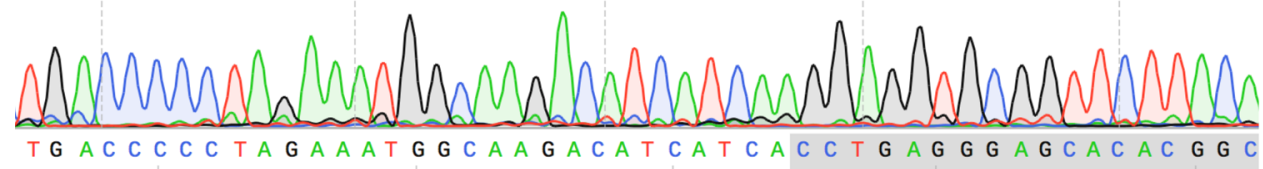

WT

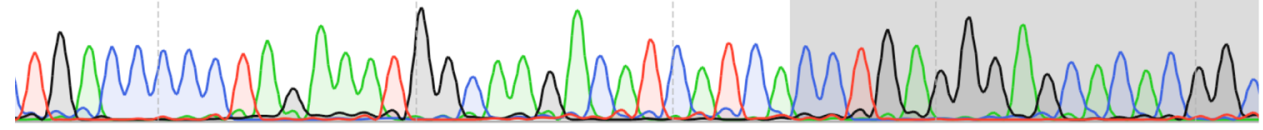

A T G G C A A G ACATCATCAA G G A G G T G C G GTTCTTACA GAAGCA C C G G C A T

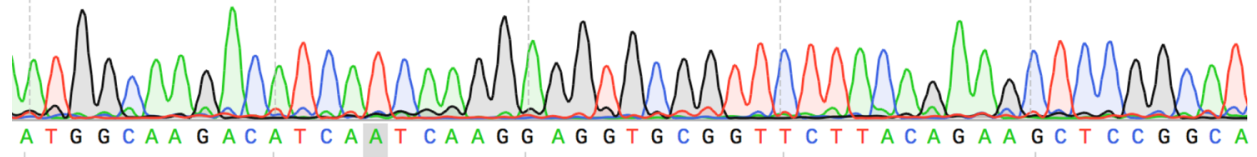

TAOK2 KO2

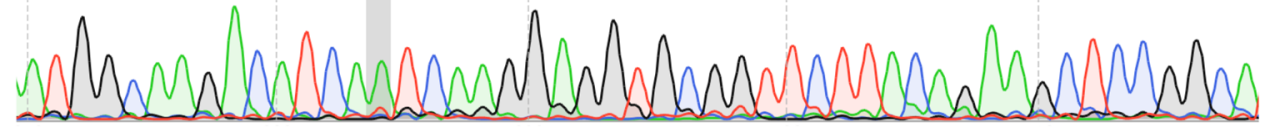


bioRxiv preprint doi: https://doi.org/10.1101/2021.04.22.440958; this version posted April 22, 2021. The copyright holder for this preprint (which was not certified by peer review) is the author/funder, who has granted bioRxiv a license to display the preprint in perpetuity. It is made available under aCC-BY-NC-ND 4.0 International license.

A.

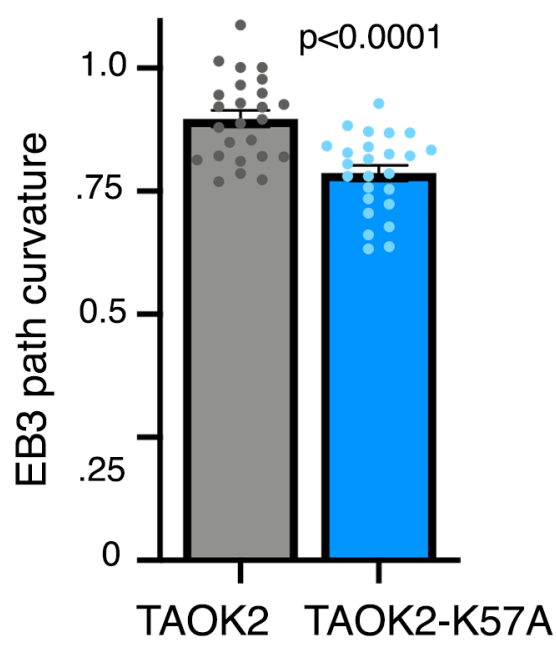

B.

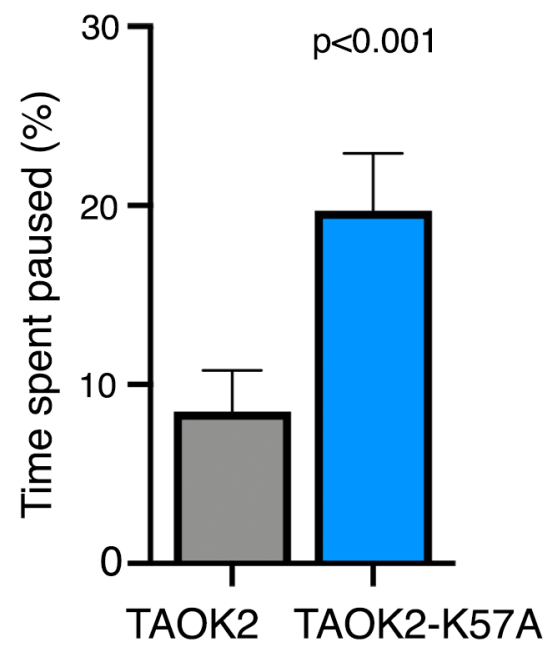

Figure S3 
TAOK2 is an ER-localized kinase that catalyzes the dynamic tethering of ER to microtubules

Kimya Nourbakhsh ${ }^{1 \#,}$ Amy A. Ferreccio ${ }^{1 \#}$, Matthew J. Bernard ${ }^{1}$ and Smita Yadav ${ }^{1 *}$

\# denotes equal contribution

1. Department of Pharmacology, University of Washington, Seattle, WA 98195

*Corresponding author, smitay@uw.edu

\section{Supplementary Information}

1. Supplementary Figures S1-S3

2. Supplementary Figure Legends

3. Movie Legends movie1-6 


\section{Supplementary Figure Legend}

Figure S1. TAOK2 localizes to the ER membrane (related to main figure 1)

(A) Confocal images of HEK293T cells stained with antibodies against calreticulin (green) and TAOK2 (magenta). Higher magnification of peripheral ER (yellow box) is shown in the bottom row. RGB profiler in ImageJ was used to plot the colocalization of calreticulin (green) and TAOK2 (red) fluorescence intensities in the region highlighted by the yellow line. Scale is $5 \mu \mathrm{m}$. (B) HEK293T cells expressing ER marker EGFP-Sec22b were fixed and stained with antibodies against TAOK2 and DAPI, scale bar is $5 \mu \mathrm{m}$. Higher magnification of peripheral ER (yellow box) is shown. RGB profiler in ImageJ was used to plot the colocalization of EGFP-Sec22b (green) and TAOK2 (red) fluorescence intensities in distinct subdomains for the region highlighted by the yellow lines. Scale bar is $5 \mu \mathrm{m}$.

Figure S2. TAOK2 C-terminal tail protein purification and generation of TAOK2 knockout cell line (related to main figure 2 and 4 )

(A) Coomassie stained gels showing eluate fractions obtained after affinity purification of GST (left) and GST-TAOK2-C (right) proteins. Fraction E4 was concentrated and used for downstream microtubule binding assays. Molecular weight proteins ladder is shown on the left.

(B) Genomic structure of TAOK2 as visualized through the UCSC genomic browser, shows the exons in gray, and region targeted by RNA guides (yellow and blue) is marked by scissors. The resulting deletion caused by genomic editing is shown in red, and premature stop codon is marked by the magenta box. 
(C) Sequence peaks show the result of DNA sequencing in wildtype and two separate KO cell lines performed after PCR of the surrounding genomic region. The change in sequence is shown in gray.

Figure S3. Effect of kinase dead TAOK2 K57A on microtubule dynamics (related to main figure 6)

(A) Microtubule directional persistence calculated as a fraction of perpendicular distance between start/end points and the length of the actual path taken is plotted for cells expressing TAOK2-WT and TAOK2-K57A. Values indicate mean \pm S.E.M., $n=10$ cells with at least 5 comet paths measured per cell, two tailed t-test.

(B) Percent total time spent by EB3 comet pausing (no growth) is plotted for TAOK2-WT and TAOK2-K57A expressing cells. Values indicate mean \pm S.E.M., $n=10$ cells with at least 5 comet paths measured per cell, two tailed t-test.

\section{Movie Legends}

\section{Supplementary Movie 1. (related to Figure 1)}

TAOK2 is an ER protein. Combined time-lapse image stacks obtained on CSU-W1 SoRa superresolution confocal microscope show representative HEK293T cell transfected with GFPTAOK2 (yellow) and ER-mRFP (magenta) to visualize the dynamics of TAOK2 on ER membranes. Image stacks were acquired every $2 \mathrm{sec}$. Movie compiled at 8 frames per sec (fps). Scale bar $=1 \mu \mathrm{m}$.

\section{Supplementary Movie 2. (related to Figure 3)}

TAOK2 is an ER-MT tether. Combined time-lapse image stacks show representative HEK293T 
cell transfected with GFP-TAOK2 and ER-mRFP. Cells were incubated with a MT binding dye (405nm excitation) for 30min prior to acquiring images on a confocal microscope. Triple channel images were acquired at a single focal plane every 2 s to simultaneously visualize TAOK2 (yellow), ER (magenta), and microtubules (cyan). Note localization of TAOK2 punctae at points of contact between ER and microtubules. Movie compiled at $8 \mathrm{fps}$. Scale bar $=2 \mu \mathrm{m}$.

\section{Supplementary Movie 3. (related to Figure 3)}

Aberrant unregulated TAOK2 tethering disrupts ER-MT dynamics. Combined time-lapse image stacks show representative HEK293T cell transfected with GFP-TAOK2(1146-1235), ER-mRFP (magenta) and stained with microtubule dye (cyan) to visualize the dynamics of ER membranes and MT growth. Complete absence of ER motility in presence of TAOK2 tether can be compared to normal ER membrane dynamics in an adjacent cell not transfected with GFPTAOK2 (1145-1235) construct. Image stacks were acquired every 2 sec. Movie compiled at 8 frames per sec (fps).

\section{Supplementary Movie 4. (related to Figure 4)}

Combined time lapse-image stacks show representative wildtype and TAOK2 knockout cell transfected with EGFP-Sec22b (cyan) and mCherry-EB3 (red) to visualize the dynamics of ER membranes and MT growth. Image stacks were acquired every $2 \mathrm{sec}$. Movie compiled at 8 frames per sec (fps).

\section{Supplementary Movie 5. (related to Figure 6)}

Combined time-lapse image stacks show representative HEK293T cell transfected with TAOK2WT, EGFP-Sec22b (magenta) and mCherry-EB3 (blue-green) to visualize the dynamics of ER membranes and MT growth. Image stacks were acquired every $2 \mathrm{sec}$. Movie compiled at 8 frames per sec (fps).

Supplementary Movie 6. (related to Figure 6) 
bioRxiv preprint doi: https://doi.org/10.1101/2021.04.22.440958; this version posted April 22, 2021. The copyright holder for this preprint (which

was not certified by peer review) is the author/funder, who has granted bioRxiv a license to display the preprint in perpetuity. It is made available under aCC-BY-NC-ND 4.0 International license.

Combined time-lapse image stacks show representative HEK293T cell transfected with TAOK2-

K57A, EGFP-Sec22b (magenta) and mCherry-EB3 (blue-green) to visualize the dynamics of ER

membranes and MT growth. Image stacks were acquired every $2 \mathrm{sec}$. Movie compiled at 8

frames per sec (fps). 